\title{
GEOGRAPHY AND THE NUMBER OF MODULI OF SURFACES OF GENERAL TYPE*
}

\author{
IGOR REIDER ${ }^{\dagger}$
}

To the memory of Andrei Nikolaevich Tyurin

\begin{abstract}
The paper considers a relationship between the Chern numbers $K_{X}^{2}, c_{2}(X)$ of a smooth minimal surface $X$ of general type and the dimension of the space of infinitesimal deformations of $X$, i.e. $h^{1}\left(\Theta_{X}\right)$, where $\Theta_{X}$ is the holomorphic tangent bundle of $X$. We prove that if the ratio of the Chern numbers $\alpha(X)=\frac{c_{2}(X)}{K_{X}^{2}} \leq \frac{3}{8}$ and $K_{X}$ is ample then

$$
h^{1}\left(\Theta_{X}\right) \leq 9\left(3 c_{2}-K^{2}\right) .
$$

On the geometric side it is shown that a smooth surface of general type $X$ with $\alpha(X) \leq \frac{3}{8}$ and $h^{1}\left(\Theta_{X}\right) \geq 3$ has two distinguished effective divisors $F$ and $E$ such that $H^{1}\left(\Theta_{X}\right)$ admits a direct sum decomposition $H^{1}\left(\Theta_{X}\right)=V_{1} \oplus V_{0}$, where $V_{1}$ is identified with a subspace of $H^{0}\left(\mathcal{O}_{X}(F)\right)$ while $V_{0}$ is identified with a subspace of $H^{0}\left(\Theta_{X} \otimes \mathcal{O}_{E}(E)\right)$. This gives a geometric interpretation of the cohomology classes in $H^{1}\left(\Theta_{X}\right)$ and allows to bound the dimension of $V_{0}$ (resp. $V_{1}$ ) in terms of geometry of the divisor $E$ (resp. $F$ ).

The main idea of the paper is to use the natural identification

$$
H^{1}\left(\Theta_{X}\right)=\operatorname{Ext}^{1}\left(\Omega_{X}, \mathcal{O}_{X}\right)
$$

where $\Omega_{X}$ is the holomorphic cotangent bundle of $X$. Then the "universal" extension gives rise to a certain vector bundle whose study constitutes the essential part of the paper.
\end{abstract}

Key words. Surfaces of general type, vector bundles, stability

AMS subject classifications. 14F05, 14J29

0. Introduction. Two questions occupy an important place in the theory of surfaces of general type: the problem of geography and the problem of moduli. Recall, if $X$ is a smooth minimal surface of general type, then its Chern numbers $K_{X}^{2}, c_{2}(X)$ are two fundamental discrete invariants of $X$. The problem of geography asks for which pairs of integers $(m, n)$ there exists a smooth minimal surface of general type $X$ with $c_{2}(X)=m$ and $K_{X}^{2}=n$. The well-known restrictions on the Chern numbers for surfaces of generaral type give us the region $P$ in $\left(c_{2}, K^{2}\right)$-plane

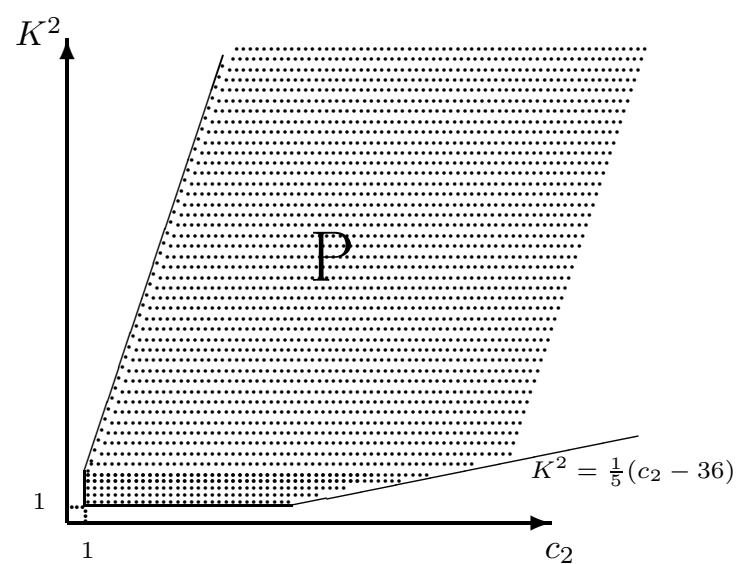

${ }^{*}$ Received December 6, 2004; accepted for publication August 12, 2005.

†Université d'Angers, Département de Mathématiques, 2, boulevard Lavoisier, 49045 ANGERS Cedex 01, France (reider@univ-angers.fr). 
where the integral points $\left(c_{2}, K^{2}\right)$ with $K^{2}+c_{2} \equiv 0(\bmod 12)$ in the shaded area are called admissible points (see [16]). The ground-braking work of U.Persson,[15], followed by the works of G.Xiao, [18],[20] and Z.Chen,[8], have shown the existence of surfaces for every admissible pair in the part of $P$ subject to $K^{2} \leq 2 c_{2}$ as well as filled the vast part of the remaining sector.

On the other hand the problem of moduli of surfaces of general type has been actively developed in the last 25 years or so (see e.g., [5],[6],[7]). In particular, F.Catanese in [6] revives a classical problem of determining an upper bound on the number of moduli of a surface of general type in terms of its Chern numbers.

The main purpose of this paper is to explore the relations between the Chern numbers $K_{X}^{2}, c_{2}(X)$ of a surface of general type $X$ and the dimension of the space of the infinitesimal deformations of the complex structure of $X$, i.e. $h^{1}\left(\Theta_{X}\right)$, where $\Theta_{X}$ is the holomorphic tangent bundle of a surface $X$.

Such relationships have been implicitly used in studying surfaces with Chern numbers close to the lower limiting line of $P$ : these surfaces and their moduli are amendable to an explicit description because they are genus 2 fibrations( see [10] and [18], [19], for general methods for studying fibered surfaces). About the other extreme of $P$ one knows from Yau's work on Kähler-Einstein metric,[21], that surfaces with $K^{2}=3 c_{2}$ are compact quotients of a unit ball in $\mathbf{C}^{2}$. This together with a result of Calabi and Vesentini, [4], yields the infinitesimal rigidity of these surfaces. One of the results of this paper is the following.

TheOREM 0.1 (= Corollary 4.10). Let $X$ be a smooth surface with $K_{X}$ ample and let $K_{X}^{2} \geq \frac{8}{3} c_{2}(X)$. Then $h^{1}\left(\Theta_{X}\right) \leq 9\left(3 c_{2}-K^{2}\right)$.

An upper bound of the above nature can be viewed as a conceptual reason for difficulties in constructing surfaces with Chern numbers which are close to the upper limiting line of $P$. Our approach also suggests where one should look for such surfaces since in deriving the above bound we give a geometric interpretation of the space of the infinitesimal deformations $H^{1}\left(\Theta_{X}\right)$. From this interpretation it follows that all such surfaces infinitesimally look as though they either come as ramified covers of some other surfaces or as divisors in a 3-fold.

To explain our approach to the study of the space $H^{1}\left(\Theta_{X}\right)$ let us consider the following hypothetical situation. Let $\pi:(\mathcal{X}, X) \longrightarrow\left(B, b_{0}\right)$ be the universal family of deformations of a smooth minimal surface of general type $X=\pi^{-1}\left(b_{0}\right)$, with the base $B$ smooth. Then the total tangent bundle $\Theta_{\mathcal{X}}$ of $\mathcal{X}$ fits into the following exact sequence

$$
0 \longrightarrow \Theta_{\mathcal{X} / B} \longrightarrow \Theta_{\mathcal{X}} \longrightarrow \pi^{*} \Theta_{B} \longrightarrow 0
$$

where $\Theta_{\mathcal{X} / B}$ is the relative tangent bundle of $\pi$. Taking the restriction of (0.1) to $X$ we obtain

$$
0 \longrightarrow \Theta_{X} \longrightarrow \Theta_{\mathcal{X}} \otimes \mathcal{O}_{X} \longrightarrow \Theta_{B, b_{0}} \otimes \mathcal{O}_{X} \longrightarrow 0
$$

By the Kodaira-Spencer theory of deformation of complex structure the coboundary map $\Theta_{B, b_{0}} \longrightarrow H^{1}\left(\Theta_{X}\right)$ arising from (0.2) is the identity. Thus the sequence (0.2) can be viewed as the element of the group of extensions $\operatorname{Ext}^{1}\left(\Theta_{B, b_{0}} \otimes \mathcal{O}_{X}, \Theta_{X}\right)$ corresponding to the identity endomorphism of $H^{1}\left(\Theta_{X}\right)$ under the natural identification $\operatorname{Ext}^{1}\left(\Theta_{B, b_{0}} \otimes \mathcal{O}_{X}, \Theta_{X}\right)=\operatorname{End}\left(H^{1}\left(\Theta_{X}\right)\right)$. Of course, such an extension can be considered independently of the geometric argument above, i.e. as long as $H^{1}\left(\Theta_{X}\right) \neq 0$ 
we have the group of extensions $\operatorname{Ext}^{1}\left(\Omega_{X}, H^{1}\left(\Theta_{X}\right)^{*} \otimes \mathcal{O}_{X}\right)=\operatorname{End}\left(H^{1}\left(\Theta_{X}\right)\right)$, where $\Omega_{X}$ is the cotangent bundle of $X$, and the identity endomorphism $i d_{H^{1}\left(\Theta_{X}\right)}$ gives rise to the following short exact sequence of sheaves on $X$

$$
0 \longrightarrow H^{1}\left(\Theta_{X}\right)^{*} \otimes \mathcal{O}_{X} \longrightarrow \mathcal{T} \longrightarrow \Omega_{X} \longrightarrow 0 .
$$

Besides its naturality the sheaf $\mathcal{T}$ is a good place to look for a relationship between $h^{1}\left(\Theta_{X}\right)$ and the Chern numbers of $X$ since the rank of $\mathcal{T}$ is $r k(\mathcal{T})=h^{1}\left(\Theta_{X}\right)+2$ and its Chern invariants are $c_{1}(\mathcal{T})=K_{X}, c_{2}(\mathcal{T})=c_{2}(X)$. A study of the sheaf $\mathcal{T}$ is the essential point of our approach.

Set $\alpha=\frac{c_{2}(X)}{K_{X}^{2}}$. The first immediate observation is that for surfaces with $\alpha<\frac{1}{2}$ (i.e. surfaces with positive index) we have a "topological" upper bound on $h^{1}\left(\Theta_{X}\right)$ coming from the semistability of $\mathcal{T}$ : if $\mathcal{T}$ is semistable with respect to some polarization on $X$ then $h^{1}\left(\Theta_{X}\right) \leq \frac{1}{1-2 \alpha}-2$.

If the above inequality fails then $\mathcal{T}$ is unstable with respect to any numerically effective nonzero divisor $D$ on $X$. This can be used to obtain some geometric information. First of all the fact that $\mathcal{T}$ is $D$-unstable implies that $\mathcal{T}$ contains the $D$-maximal destabilizing subsheaf $\mathcal{T}_{1}^{D}$ which gives rise to a nontrivial decomposition of the canonical divisor of $X$

$$
K_{X}=L_{1}+E_{1}
$$

where $L_{1}=c_{1}\left(\mathcal{T}_{1}^{D}\right)$ and $E_{1}=c_{1}\left(\mathcal{T} / \mathcal{T}_{1}^{D}\right)$. This decomposition becomes especially meaningful geometrically once we take $D=K_{X}$ and $\alpha \leq \frac{3}{8}$. We show that in this case $L_{1}$ is in the positive cone of the Néron-Severi group $N S(X)$ of $X$ and $E_{1}$ is an effective nonzero divisor whose degree with respect to $K_{X}$ is bounded by a function depending on $\left(3 c_{2}-K^{2}\right)$ (see Corollary 1.7). Furthermore, the rank of $K_{X}$-maximal destabilizing subsheaf $\mathcal{T}_{1}$ turns out to be 2 or 3 and the inclusion $\mathcal{T}_{1} \longrightarrow \mathcal{T}$ combined with the defining sequence (0.3) gives rise to a generically surjective morphism

$$
\mu_{1}: \mathcal{T}_{1} \longrightarrow \Omega_{X}
$$

This morphism looks as if it were the codifferential of a morphism $f: X \longrightarrow Y$ which is generically of maximal rank and where $\operatorname{dim} Y=2$ or 3 . Of course, there is no reason for $\mu_{1}$ to come from such a geometric situation. However, it gives rise to a decomposition of $H^{1}\left(\Theta_{X}\right)$ as a direct sum of its subspaces $H^{1}\left(\Theta_{X}\right)=V_{0} \oplus V_{1}$. Each of these subspaces has features characteristic to the aforementioned geometric situation (see Proposition 2.2):

1. $V_{0}$ is a subspace of $H^{1}\left(\Theta_{X}\right)$ contained in the kernel of the obvious morphism

$$
H^{1}\left(\Theta_{X}\right) \longrightarrow H^{1}\left(\Theta_{X}(E)\right)
$$

for some component $E$ of $E_{1}$ in the decomposition (0.4), i.e. it looks as though $E$ is the ramification divisor of some morphism of $X$ onto another surface;

2. $V_{1}$ injects into $H^{0}\left(\mathcal{O}_{X}(F)\right)$, where $F$ is again a component of $E_{1}$, i.e. $V_{1}$ looks as a subspace of infinitesimal deformations of a divisor in a 3 -fold.

The above result gives a geometric interpretation of the cohomology classes in $H^{1}\left(\Theta_{X}\right)$ as global sections of $\mathcal{O}_{X}(F)$ or $\Theta_{X} \otimes \mathcal{O}_{E}(E)$. It also allows to obtain upper bounds on the dimensions of the subspaces $V_{0}$ and $V_{1}$ in terms of geometry 
of the divisors $E$ and $F$ respectively (see e.g., Corollary 2.4, Corollary 3.9). Putting these bounds together with the estimate of $E_{1} \cdot K_{X}$ as a function of $\left(3 c_{2}-K^{2}\right)$ we derive the upper bound for $h^{1}\left(\Theta_{X}\right)$ as a function of $\left(3 c_{2}-K^{2}\right)$ (see Corollary 4.10). We also point out that the nature of the bound as a linear function of $\left(3 c_{2}-K^{2}\right)$ can not be improved in view of examples which we discuss in Example 4.11.

The paper is organized as follows.

In $\S 1$ we define the extension bundle $\mathcal{T}$ as in $(0.3)$ and consider its properties from the point of view of stability.

In $\S 2$ we introduce the notion of divisorial and locally supported moduli and show that surfaces with $\alpha=\frac{c_{2}(X)}{K_{X}^{2}} \leq \frac{3}{8}$ and $h^{1}\left(\Theta_{X}\right)>2$ have the property that the space of the infinitesimal deformations $H^{1}\left(\Theta_{X}\right)$ admits a vector space decomposition $H^{1}\left(\Theta_{X}\right)=V_{0} \oplus V_{1}$ where $V_{1}$ is divisorial moduli and $V_{0}$ is locally supported moduli of $X$. We also derive an upper bound on the dimension of the divisorial moduli $V_{1}$ of $X$ (Corollary 2.4).

The sections $\S 3$ and $\S 4$ are devoted to a study of the subspace $V_{0}$ of locally supported moduli and, particularly, to a study of the divisor on which $V_{0}$ is supported.

In section $\S 5$ we consider surfaces whose Chern numbers are subject to $3 c_{2}-K^{2} \leq \frac{1}{2} \sqrt{K^{2}}$. This "quadratic" condition emerges naturally in view of the bound on the degree of $E_{1}$ obtained in Corollary 1.7. The point is that the Hodge index together with the "quadratic" condition implies that the intersection form restricted to the sublattice of $N S(X)$ generated by the irreducible components of $E_{1}$ is negative semidefinite. This allows us to give a detailed description of these components (Lemma 5.4) as well as to deduce conditions for these surfaces to be fibred by curves of genus $\leq\left(3 c_{2}-K^{2}\right)$.

1. Extension construction. Let $X$ be a smooth minimal surface of general type. The holomorphic tangent (resp. cotangent) bundle of $X$ will be denoted by $\Theta_{X}$ (resp. $\Omega_{X}$ ). Throughout the paper, unless said otherwise, we assume $H^{1}\left(\Theta_{X}\right) \neq 0$. For a nonzero subspace $V$ of $H^{1}\left(\Theta_{X}\right)$ we consider the extension

$$
0 \longrightarrow V^{*} \otimes \mathcal{O}_{X} \longrightarrow \mathcal{T}_{V} \longrightarrow \Omega_{X} \longrightarrow 0
$$

corresponding to the natural inclusion $V \subset H^{1}\left(\Theta_{X}\right)$ where the following natural identifications are used:

$$
\operatorname{Ext}^{1}\left(\Omega_{X}, V^{*} \otimes \mathcal{O}_{X}\right)=V^{*} \otimes H^{1}\left(\Theta_{X}\right)=H o m_{\mathbf{C}}\left(V, H^{1}\left(\Theta_{X}\right)\right) .
$$

We will often refer to the sheaf $\mathcal{T}_{V}$ sitting in the middle of (1.1) as extension corresponding to $V$. If $V=H^{1}\left(\Theta_{X}\right)$, then the corresponding sheaf will be denoted by $\mathcal{T}$.

The invariants of $\mathcal{T}$ are easily computed from the defining sequence (1.1):

$$
r k(\mathcal{T})=h^{1}\left(\Theta_{X}\right)+2, \quad c_{1}(\mathcal{T})=c_{1}\left(\Omega_{X}\right)=K_{X}, \quad c_{2}(\mathcal{T})=c_{2}\left(\Omega_{X}\right)=c_{2}(X) .
$$

If no ambiguity is likely we will omit $X$ in the above notation. Set $\alpha=\frac{c_{2}(X)}{K_{X}^{2}}$ and assume $\alpha<\frac{1}{2}$. The semistability of $\mathcal{T}$ gives "the topological" upper bound for $h^{1}\left(\Theta_{X}\right)$ as a function of $\alpha$.

Proposition 1.1. Let $\alpha<\frac{1}{2}$. If $\mathcal{T}$ is semistable with respect to some ample divisor $H$ on $X$ then $h^{1}\left(\Theta_{X}\right) \leq \frac{1}{1-2 \alpha}-2$. 
Proof. The Bogomolov-Gieseker inequality (see, e.g., [13]) applied to $\mathcal{T}$ gives

$$
2\left(h^{1}\left(\Theta_{X}\right)+2\right) c_{2} \geq\left(h^{1}\left(\Theta_{X}\right)+1\right) K_{X}^{2} \Leftrightarrow 2\left(h^{1}\left(\Theta_{X}\right)+2\right) \alpha \geq h^{1}\left(\Theta_{X}\right)+1 .
$$

Solving for $h^{1}\left(\Theta_{X}\right)$ yields the asserted inequality.

From now on we assume that $\alpha<\frac{1}{2}$ and $h^{1}\left(\Theta_{X}\right)>\frac{1}{1-2 \alpha}-2$. In view of Proposition 1.1 the vector bundle $\mathcal{T}$ is unstable with respect to any numerically effective (nef) divisor $D$ on $X$. We consider $D$-destabilizing filtration of $\mathcal{T}$ for $D$ nef and big $\left(D^{2}>0\right)$ :

$$
\mathcal{T}=\mathcal{T}_{s}^{D} \supset \cdots \supset \mathcal{T}_{1}^{D} \supset \mathcal{T}_{0}^{D}=0 .
$$

Associated to this filtration we have:

$$
\mathcal{G}_{i}^{D}=\mathcal{T}_{i}^{D} / \mathcal{T}_{i-1}^{D}, L_{i}=c_{1}\left(\mathcal{G}_{i}^{D}\right), d_{i}=c_{2}\left(\mathcal{G}_{i}^{D}\right), r_{i}=r k\left(\mathcal{G}_{i}^{D}\right), \alpha_{i}=\frac{L_{i} . D}{r_{i} K . D} .
$$

The main properties of the filtration (1.2) are (see [13]):

- the graded sheaves $\mathcal{G}_{i}^{D}$ are $D$-semistable,

- $\alpha_{1}>\ldots>\alpha_{s}$

The subsheaf $\mathcal{T}_{1}^{D}=\mathcal{G}_{1}^{D}$ is called the $D$-maximal destabilizing subsheaf of $\mathcal{T}$.

Following Miyaoka,[13], we have the notion of semipositivity of a torsion-free sheaf.

DEFINITION 1.2 .

a). A torsion-free sheaf $\mathcal{F}$ on $X$ is called semipositive with respect to a nef divisor $D$ (also referred to as D-semipositive) if for any torsion-free quotient $\mathcal{Q}$ of $\mathcal{F}$ one has $c_{1}(\mathcal{Q}) . D \geq 0$.

b). $\mathcal{F}$ is called generically semipositive if it is so for any nef divisor on $X$.

LEMma 1.3. The sheaf $\mathcal{T}_{V}$ is generically semipositive.

Proof. Suppose $\mathcal{T}_{V}$ is not $D$-semipositive. Then there exists a subsheaf $\mathcal{G}$ of $\mathcal{T}$ whose quotient $\mathcal{Q}$ is torsion-free and $c_{1}(\mathcal{Q}) . D<0$ or, equivalently, $c_{1}(\mathcal{G}) . D>K . D$. Consider the diagram

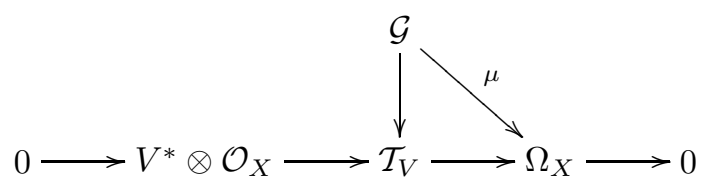

The induced morphism $\mu: \mathcal{G} \longrightarrow \Omega_{X}$ must be generically of maximal rank, since otherwise $\operatorname{Im}(\mu)$ is a subsheaf of $\Omega_{X}$ such that $c_{1}(\operatorname{Im}(\mu)) . D \geq c_{1}(\mathcal{G}) . K>K . D$ contradicting generic semipositivity of $\Omega_{X}$. In particular, the rank $r=r k(\mathcal{G}) \geq 2$. Taking the $r$-th exterior power of (1.4) we obtain

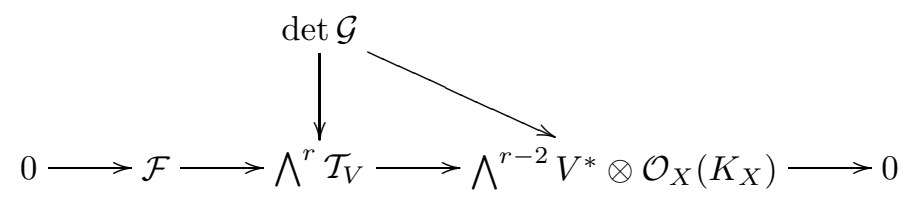


The slanted arrow in $(1.5)$ must be zero since $c_{1}(\mathcal{G}) . D>K . D$. This yields a nonzero morphism $\operatorname{det} \mathcal{G} \longrightarrow \mathcal{F}$. But $\mathcal{F}$ fits into the following exact sequence

$$
0 \longrightarrow \bigwedge^{r} V^{*} \otimes \mathcal{O}_{X} \longrightarrow \mathcal{F} \longrightarrow \bigwedge^{r-1} V^{*} \otimes \Omega_{X} \longrightarrow 0
$$

which gives a nonzero morphism $\operatorname{det} \mathcal{G} \longrightarrow \Omega_{X}$ contradicting generic semipositivity of $\Omega_{X}$ प

Using the $D$-destabilizing filtration (1.2) and the notation in (1.3) we obtain

$$
2 c_{2}=2 \sum_{i} d_{i}+2 \sum_{i<j} L_{i} \cdot L_{j}=2 \sum_{i} d_{i}+K^{2}-\sum_{i} L_{i}^{2}
$$

Since $\mathcal{G}_{i}$ 's are $D$-semistable the Bogomolov-Gieseker inequality gives $2 d_{i} \geq \frac{r_{i}-1}{r_{i}} L_{i}^{2}$. Substituting into (1.6) we obtain

$$
2 c_{2} \geq 2 d_{1}-L_{1}^{2}-\sum_{i \geq 2} \frac{1}{r_{i}} L_{i}^{2}+K^{2}
$$

By Hodge Index $L_{i}^{2} \leq \frac{\left(L_{i} \cdot D\right)^{2}}{D^{2}}$. This gives

$$
\frac{1}{r_{i}} L_{i}^{2} \leq \frac{L_{i} \cdot D}{r_{i}} \frac{L_{i} \cdot D}{D^{2}}=\alpha_{i} \frac{L_{i} \cdot D}{D^{2}}(K . D) .
$$

Substituting in (1.7)

$$
2 c_{2} \geq 2 d_{1}-L_{1}^{2}-\left(\sum_{i \geq 2} \alpha_{i} L_{i} . D\right) \frac{K . D}{D^{2}}+K^{2} .
$$

REMARK 1.4. From Lemma 1.3 it follows that $\alpha_{s} \geq 0$. This implies that $\alpha_{i}, L_{i} . D \geq 0$ for all $i$ and the inequality is strict for $1 \leq i<s$.

From now on we assume $D=K_{X}$. Substituting this in (1.8) we obtain

$$
2 c_{2} \geq 2 d_{1}-L_{1}^{2}-\sum_{i \geq 2} \alpha_{i} L_{i} \cdot K+K^{2}
$$

LEMMA 1.5. If $L_{1}^{2} \leq 0$, then $\alpha>\frac{1}{2}-\frac{1}{8 r_{1}}$.

Proof. The Bogomolov-Gieseker inequality for $\mathcal{G}_{1}$ together with $L_{1}^{2} \leq 0$ yields

$$
2 c_{2} \geq-\sum_{i \geq 2} \alpha_{i} L_{i} \cdot K+K^{2}>-\alpha_{1}\left(K^{2}-L_{1} \cdot K\right)+K^{2}=-\alpha_{1}\left(1-r_{1} \alpha_{1}\right) K^{2}+K^{2}
$$

where the second inequality follows from Remark 1.4 and the fact that the sequence $\left\{\alpha_{i}\right\}$ is strictly decreasing. Dividing by $K^{2}$ we obtain

$$
2 \alpha>-\alpha_{1}\left(1-r_{1} \alpha_{1}\right)+1
$$


or, equivalently,

$$
r_{1} \alpha_{1}^{2}-\alpha_{1}+(1-2 \alpha)<0
$$

In particular, the discriminant of the quadratic polynomial on the left-hand side must be positive:

$$
1-4 r_{1}(1-2 \alpha)>0 \Leftrightarrow \alpha>\frac{1}{2}-\frac{1}{8 r_{1}} .
$$

$\square$

Proposition 1.6. If $\alpha \leq \frac{3}{8}$, then $L_{1}^{2}>0$ and the morphism $\mu_{1}: \mathcal{T}_{1} \longrightarrow \Omega_{X}$ induced from

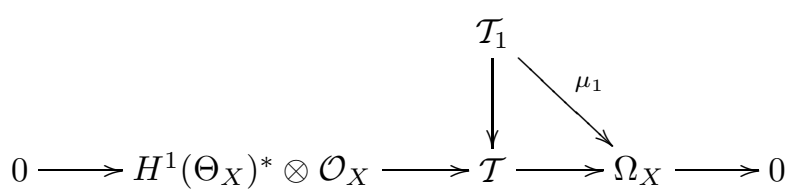

is generically surjective. In particular, the rank $r_{1}$ of $\mathcal{T}_{1}$ is 2 or 3 .

Proof. From Lemma 1.5 it follows that $L_{1}^{2}>0$. Since $\Omega_{X}$ can not have subsheaves of rank 1 of D-dimension 2 we deduce that $r_{1} \geq 2$ and $\mu_{1}$ is generically surjective. To obtain an upper bound on $r_{1}$ we use (1.8)

$$
2 c_{2} \geq-\alpha_{1} K^{2}+K^{2} \Leftrightarrow r_{1}<\frac{1}{1-2 \alpha} \leq 4 .
$$

प

Corollary 1.7. If $\alpha \leq \frac{3}{8}$ and $h^{1}\left(\Theta_{X}\right)>2$ then $K_{X}$ has a distinguished decomposition $K_{X}=L_{1}+E_{1}$ where $L_{1}=c_{1}\left(\mathcal{T}_{1}\right)$ is in the positive cone $C^{+}(X)$ of the Néron-Severi group $N S(X)$ of $X$ and $E_{1}$ is an effective nonzero divisor. Furthermore, the rank $r_{1}$ of $\mathcal{T}_{1}$ is equal to 2 or 3 and

$$
\frac{E_{1} \cdot K}{K^{2}}<\left\{\begin{array}{l}
2(3 \alpha-1), \text { if } r_{1}=3 \\
\frac{8(3 \alpha-1)}{1+\sqrt{1+16(3 \alpha-1)}}, \text { if } r_{1}=2
\end{array} .\right.
$$

Proof. If $\alpha \leq \frac{3}{8}$ then the "topological" bound $\frac{1}{1-2 \alpha}-2 \leq 2$. So the BogomolovGieseker inequality for $\mathcal{T}$ fails as soon as $h^{1}\left(\Theta_{X}\right)>2$. In particular, we have the $K_{X}$-maximal destabilizing subsheaf $\mathcal{T}_{1}$ which is subject to Proposition 1.6. Hence the assertion about $L_{1}$ and $r_{1}$. To see the properties of $E_{1}$ we consider the diagram (1.10) according the values of $r_{1}$. 
1) $\mathbf{r}_{1}=\mathbf{3}$ : in this case the above mentioned diagram has the following form

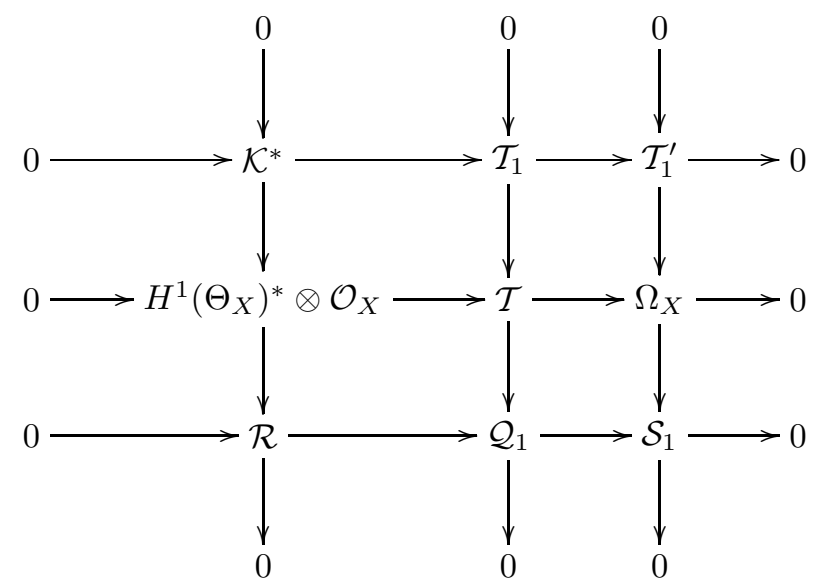

where $\mathcal{T}_{1}^{\prime}=I m \mu_{1}, \mathcal{K}^{*}=\operatorname{ker} \mu_{1}, \mathcal{S}_{1}=\operatorname{coker}_{1}, \quad \mathcal{Q}_{1}=\mathcal{T} / \mathcal{T}_{1}, \mathcal{R}=\left(H^{1}\left(\Theta_{X}\right)^{*} \otimes\right.$ $\left.\mathcal{O}_{X}\right) / \mathcal{K}^{*}$. From (1.11) we obtain that $\mathcal{K}^{*}$ is a line bundle whose dual $\mathcal{K}$ is generated by global sections outside of a subscheme of codim $\geq 2$. More precisely, dualizing the column on the left we obtain

$$
0 \longrightarrow \mathcal{R}^{*} \longrightarrow H^{1}\left(\Theta_{X}\right) \otimes \mathcal{O}_{X} \longrightarrow \mathcal{K} \longrightarrow \mathcal{E} x t^{1}\left(\mathcal{R}, \mathcal{O}_{X}\right) \longrightarrow 0
$$

where the sheaf $\mathcal{E} x t^{1}\left(\mathcal{R}, \mathcal{O}_{X}\right)$ is supported on a subscheme of codim $\geq 2$. Putting $F=$ $c_{1}(\mathcal{K})$ we conclude that $F$ is an effective divisor and $L_{1}=c_{1}\left(\mathcal{T}_{1}^{\prime}\right)-F=K_{X}-c_{1}\left(\mathcal{S}_{1}\right)-F$. This implies

$$
E_{1}=K_{X}-L_{1}=c_{1}\left(\mathcal{S}_{1}\right)+F
$$

Since both $c_{1}\left(\mathcal{S}_{1}\right)$ and $F$ are effective and they can not vanish simultaneously we obtain that $E_{1}$ is an effective nonzero divisor.

To obtain the asserted upper bound on the degree (with respect to $K_{X}$ ) of $E_{1}$ we use (1.8) to obtain

$$
2 c_{2}>-\alpha_{1} K^{2}+K^{2} \Leftrightarrow \frac{L_{1} \cdot K}{3 K^{2}}>1-2 \alpha \Leftrightarrow \frac{E_{1} \cdot K}{K^{2}}<2(3 \alpha-1) .
$$


2) $\mathbf{r}_{\mathbf{1}}=\mathbf{2}:$ the diagram (1.10) has the following form

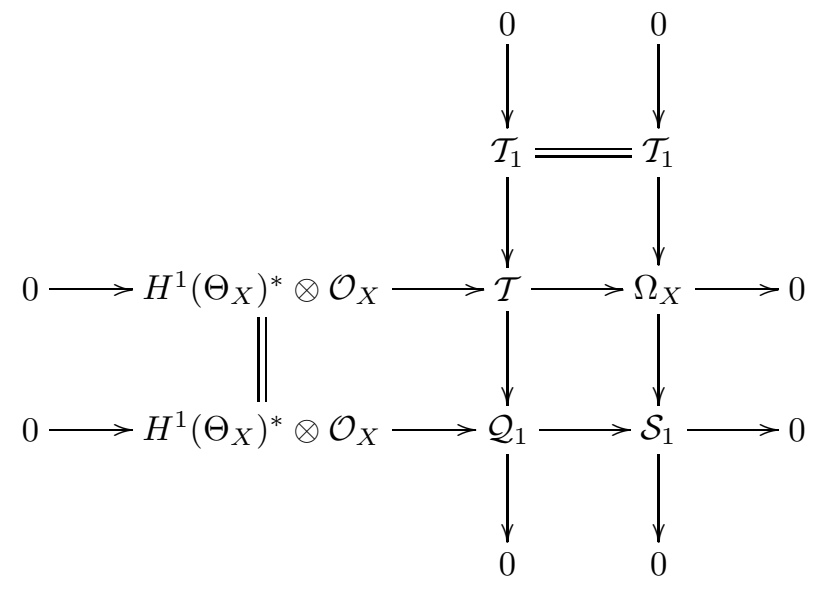

This implies that $E_{1}=K_{X}-L_{1}=c_{1}\left(\mathcal{S}_{1}\right)$ is effective and it must be nonzero (otherwise the extension defining $\mathcal{T}$ splits). To bound the degree of $E_{1}$ in this case we use a result of Miyaoka which says that $3 d_{1}=3 c_{2}\left(\mathcal{T}_{1}\right) \geq c_{1}^{2}\left(\mathcal{T}_{1}\right)=L_{1}^{2}$ (see Remark 4.18,[12]). Substituting this in (1.8) we obtain

$$
\begin{aligned}
2 c_{2} \geq-\frac{1}{3} L_{1}^{2}- & \sum_{i \geq 2} \alpha_{i} K \cdot L_{i}+K^{2}>-\frac{1}{3} L_{1}^{2}-\alpha_{1} E_{1} \cdot K+K^{2} \\
& =-\frac{1}{3}\left(K-E_{1}\right)^{2}-\frac{K^{2}-E_{1} \cdot K}{2 K^{2}} E_{1} \cdot K+K^{2} .
\end{aligned}
$$

Dividing by $K^{2}$ and using the Hodge Index $\frac{E_{1}^{2}}{K^{2}} \leq\left(\frac{E_{1} \cdot K}{K^{2}}\right)^{2}$ one obtains the following inequality

$$
\left(\frac{E_{1} \cdot K}{K^{2}}\right)^{2}+\frac{E_{1} \cdot K}{K^{2}}-4(3 \alpha-1)<0
$$

Solving it for $\frac{E_{1} \cdot K}{K^{2}}$ yields

$$
\frac{E_{1} \cdot K}{K^{2}}<\frac{-1+\sqrt{1+16(3 \alpha-1)}}{2}=\frac{8(3 \alpha-1)}{1+\sqrt{1+16(3 \alpha-1)}} .
$$

2. Two types of infinitesimal deformations. The situation encountered in the proof of Corollary 1.7 looks as though our surface $X$ admits a morphism $f$ : $X \longrightarrow Y$ which is generically of maximal rank and where $\operatorname{dim} Y=r_{1}=2$ or 3 . With this hypothetical geometric interpretation in mind one could say that the case $r_{1}=2$ corresponds to the situation where all infinitesimal deformations of $X$ come from the infinitesimal deformations of the ramification divisor of $f$ and the case $r_{1}=3$ would generally brake into two parts: the infinitesimal deformations of the divisor $X^{\prime}=\operatorname{Im}(f)$ in the 3 -fold $Y$ and the infinitesimal deformations of the ramification divisor of $f$. Of course, there is no reason for the morphism $\mu_{1}: \mathcal{T}_{1} \longrightarrow \Omega_{X}$ in (1.10) 
to come from geometry. However, the infinitesimal deformations of $X$ have all the features of such geometric situations: in the case $r_{1}=2$ all elements of $H^{1}\left(\Theta_{X}\right)$ are supported on the divisor $E_{1}$ as in Corollary 1.7 and in the case $r_{1}=3$ we can brake $H^{1}\left(\Theta_{X}\right)$ into two parts: $V_{0}=H^{0}\left(\mathcal{R}^{*}\right)$ (see (1.12)) and $V_{1}$, a subspace of $H^{1}\left(\Theta_{X}\right)$ complementary to $V_{0}$. The subspace $V_{0}$ is as in the case $r_{1}=2$ while the subspace $V_{1}$ injects into $H^{0}(X, \mathcal{K})$ (see $(1.11)$ for notation), i.e. it looks like infinitesimal deformations of a divisor in a 3 -fold.

The following definition is motivated by the above discussion.

Definition 2.1. 1) A subspace $V$ in $H^{1}\left(\Theta_{X}\right)$ is called a divisorial moduli of $X$ if there exists a divisor $D$ on $X$ and an injective linear map $V \longrightarrow H^{0}\left(\mathcal{O}_{X}(D)\right)$. 2) A subspace $V$ in $H^{1}\left(\Theta_{X}\right)$ is called a locally supported moduli of $X$ if there exists a nonzero effective divisor $E$ on $X$ such that the sequence

$$
0 \longrightarrow H^{0}\left(\Theta_{X} \otimes \mathcal{O}_{E}(E)\right) \longrightarrow H^{1}\left(\Theta_{X}\right) \stackrel{e}{\longrightarrow} H^{1}\left(\Theta_{X}(E)\right)
$$

is exact and $V \subset \operatorname{ker}(e)$, where $e$ is a section defining $E$. In this case we will say that $V$ is locally supported on the divisor $E$.

3) We say that $H^{1}\left(\Theta_{X}\right)$ admits a decomposition into divisorial and locally supported moduli if there exists a vector space decomposition $H^{1}\left(\Theta_{X}\right)=V \oplus V^{\prime}$ such that $V$ (resp. $\left.V^{\prime}\right)$ is a divisorial (resp. locally supported) moduli.

If $V=0$ (resp. $\left.V^{\prime}=0\right)$, we say that $X$ has locally supported (resp. divisorial) moduli only.

Let us show that the space of the infinitesimal deformations of $X$ subject to the conditions of Corollary 1.7 admits a decomposition into divisorial and locally supported moduli. In order to do this we return to (1.12) and consider the decomposition

$$
H^{1}\left(\Theta_{X}\right)=V_{0} \oplus V_{1}
$$

where we put $V_{0}=H^{0}\left(\mathcal{R}^{*}\right)$ and $V_{1}$, a subspace of $H^{1}\left(\Theta_{X}\right)$ complementary to $V_{0}$.

Proposition 2.2. The decomposition (2.1) is a decomposition into divisorial and locally supported moduli. The subspace $V_{1}$ injects into $H^{0}\left(\mathcal{O}_{X}(F)\right)$ (recall: $F=c_{1}(\mathcal{K})$ ) and $V_{0}$ is locally supported on $E^{\prime}=c_{1}\left(\mathcal{S}_{1}\right)$, i.e. $V_{0}$ injects into $H^{0}\left(\Theta_{X} \otimes \mathcal{O}_{E^{\prime}}\left(E^{\prime}\right)\right)$. Furthermore, if $r_{1}=2$ then $X$ has locally supported moduli only, and if $r_{1}=3$ and $V_{0}=H^{0}\left(\mathcal{R}^{*}\right)=0$ then $X$ has divisorial moduli only.

Proof. The injection $V_{1} \longrightarrow H^{0}\left(\mathcal{O}_{X}(F)\right)$ follows from the definition of $V_{1}$ and (1.12). To see the assertion about $V_{0}$ we assume it to be nonzero and consider the extension of $\Omega_{X}$ corresponding to the natural inclusion $V_{0} \subset H^{1}\left(\Theta_{X}\right)$

$$
0 \longrightarrow V_{0}^{*} \otimes \mathcal{O}_{X} \longrightarrow \mathcal{T}_{V_{0}} \longrightarrow \Omega_{X} \longrightarrow 0 .
$$

The dual of the bottom sequence (1.11) implies that $V_{0}$ injects into $H^{0}\left(\mathcal{E} x t^{1}\left(\mathcal{S}_{1}, \mathcal{O}_{X}\right)\right)$. The latter space is contained in the kernel of $H^{1}\left(\Theta_{X}\right) \longrightarrow H^{1}\left(\mathcal{T}_{1}^{\prime *}\right)$ (this is seen by taking the dual of the column on the right-hand side of (1.11)). From this it follows that the morphism $\left(\mathcal{T}_{1}^{\prime}\right)^{* *} \longrightarrow \Omega_{X}$ induced by $\mathcal{T}_{1}^{\prime} \longrightarrow \Omega_{X}$ in (1.11) lifts to a morphism to $\mathcal{T}_{V_{0}}$, i.e. we have

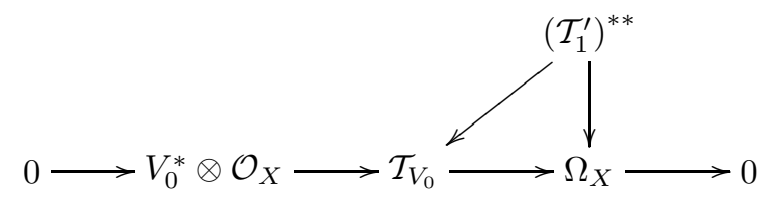


Factoring out by the torsion part of $\mathcal{T}_{V_{0}} /\left(\mathcal{T}_{1}^{\prime}\right)^{* *}$ we arrive to the following situation

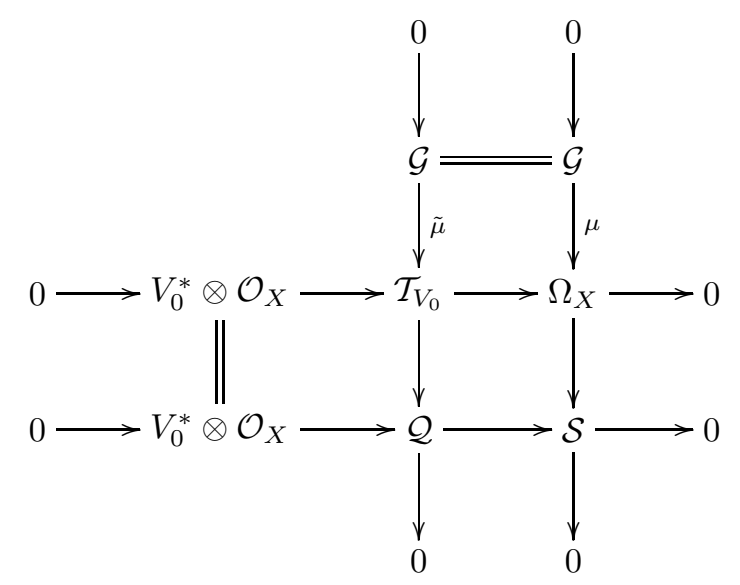

where $\mathcal{G}$ is a locally free subsheaf of $\Omega_{X}$ and $\mathcal{Q}$ is torsion-free. Let $L=c_{1}(\mathcal{G})$ and $E=c_{1}(\mathcal{S})$. So $E$ is effective nonzero divisor. This divisor is related to $E_{1}$ in (1.13) and $E^{\prime}$ as follows

$$
E_{1}=F+c_{1}\left(\mathcal{S}_{1}\right)=F+E^{\prime}=F+c_{1}\left(\operatorname{Tor}\left(\mathcal{T}_{V_{0}} /\left(\mathcal{T}_{1}^{\prime}\right)^{* *}\right)\right)+E
$$

In particular, $E$ is a component of $E^{\prime}$. We will show that the subspace $V_{0}$ is locally supported on $E$ and hence on $E^{\prime}$ as asserted in the proposition.

Let $e$ be a global section of $\mathcal{O}_{X}(E)$ defining $E$ and consider the short exact sequence

$$
0 \longrightarrow \Theta_{X} \stackrel{e}{\longrightarrow} \Theta_{X}(E) \longrightarrow \Theta_{X} \otimes \mathcal{O}_{E}(E) \longrightarrow 0 .
$$

Observe that $\Theta_{X}(E)=\Omega_{X}(-L)$. Since $L=c_{1}(\mathcal{G})=c_{1}\left(\left(\mathcal{T}_{1}^{\prime}\right)^{* *}\right)+$ $c_{1}\left(\operatorname{Tor}\left(\mathcal{T}_{V_{0}} /\left(\mathcal{T}_{1}^{\prime}\right)^{* *}\right)\right)=L_{1}+F+c_{1}\left(\operatorname{Tor}\left(\mathcal{T}_{V_{0}} /\left(\mathcal{T}_{1}^{\prime}\right)^{* *}\right)\right)$ we have that $L$ has D-dimension 2. This implies that $H^{0}\left(\Theta_{X}(E)\right)=0$ and the sequence

$$
0 \longrightarrow H^{0}\left(\Theta_{X} \otimes \mathcal{O}_{E}(E)\right) \longrightarrow H^{1}\left(\Theta_{X}\right) \stackrel{e}{\longrightarrow} H^{1}\left(\Theta_{X}(E)\right)
$$

is exact (the same argument holds for $E^{\prime}$ as well). It remains to check that $V_{0}$ is contained in the kernel of $H^{1}\left(\Theta_{X}\right) \stackrel{e}{\longrightarrow} H^{1}\left(\Theta_{X}(E)\right)$. This can be seen by taking the second exterior power of $(2.2)$ and tensoring it with $\mathcal{O}_{X}(-L)$

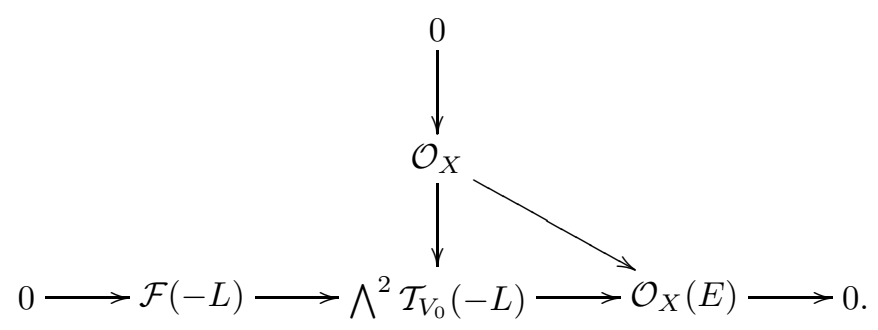

The sheaf $\mathcal{F}(-L)$ fits into the following exact sequence

$$
0 \longrightarrow \bigwedge^{2} V_{0}^{*} \otimes \mathcal{O}_{X}(-L) \longrightarrow \mathcal{F}(-L) \longrightarrow V_{0}^{*} \otimes \Theta_{X}(E) \longrightarrow 0
$$


From (2.4) we deduce that $e \in H^{0}\left(\mathcal{O}_{X}(E)\right)$ lies in the kernel of the coboundary morphism

$$
H^{0}\left(\mathcal{O}_{X}(E)\right) \longrightarrow H^{1}(\mathcal{F}(-L))
$$

This morphism and (2.5) give the linear map

$$
H^{0}\left(\mathcal{O}_{X}(E)\right) \longrightarrow V_{0}^{*} \otimes H^{1}\left(\Theta_{X}(E)\right)=H o m\left(V_{0}, H^{1}\left(\Theta_{X}(E)\right)\right)
$$

which is induced by the obvious cup-product

$$
H^{0}\left(\mathcal{O}_{X}(E)\right) \otimes H^{1}\left(\Theta_{X}\right) \longrightarrow H^{1}\left(\Theta_{X}(E)\right) .
$$

Since $e$ is mapped to zero in (2.6) we deduce that $V_{0}$ is contained in $\operatorname{ker}\left(H^{1}\left(\Theta_{X}\right) \stackrel{e}{\longrightarrow}\right.$ $\left.H^{1}\left(\Theta_{X}(E)\right)\right)$.

REMARK 2.3. From Proposition 2.2 it follows that the inclusion $V_{0} \subset H^{1}\left(\Theta_{X}\right)$ factors through $H^{0}\left(\Theta_{X} \otimes \mathcal{O}_{E}(E)\right)$ as follows

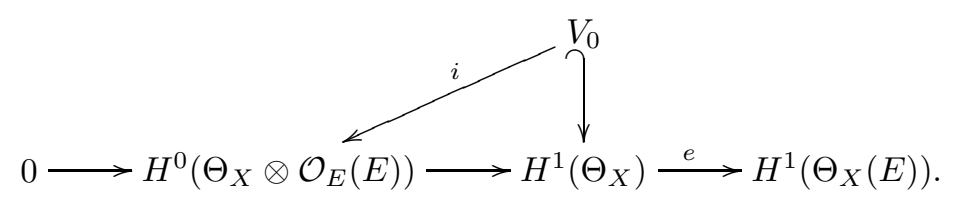

In fact one can be more precise. First remark that the fact that $V_{0}$ is annihilated by $e$ implies that the morphism $\Omega_{X}(-E) \longrightarrow \Omega_{X}$ lifts to $\mathcal{T}_{V_{0}}$ and this lift $\Omega_{X}(-E) \longrightarrow \mathcal{T}_{V_{0}}$ factors through $\mathcal{G}$ in (2.2). This gives the following diagram

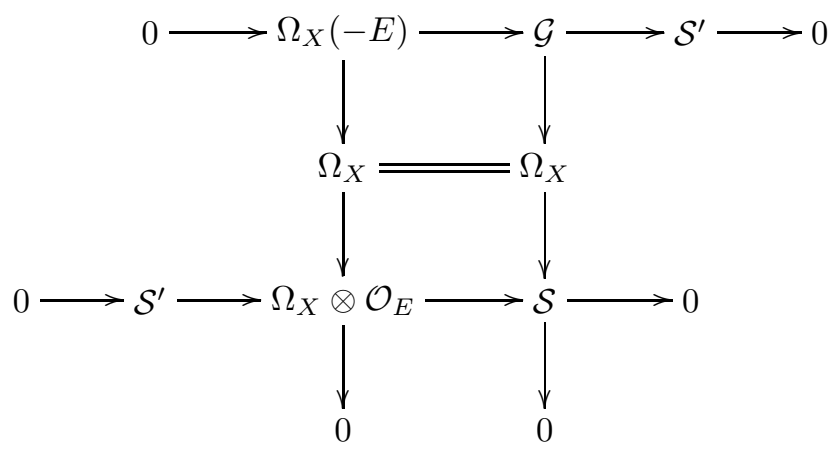

Dualizing the bottom sequence we obtain

$$
0 \longrightarrow \mathcal{E} x t^{1}\left(\mathcal{S}, \mathcal{O}_{X}\right) \longrightarrow \Theta_{X} \otimes \mathcal{O}_{E}(E) \longrightarrow \mathcal{E} x t^{1}\left(\mathcal{S}^{\prime}, \mathcal{O}_{X}\right) \longrightarrow 0
$$

and the injection $V_{0} \stackrel{i}{\longrightarrow} H^{0}\left(\Theta_{X} \otimes \mathcal{O}_{E}(E)\right)$ factors through $H^{0}\left(\mathcal{E} x t^{1}\left(\mathcal{S}, \mathcal{O}_{X}\right)\right)$

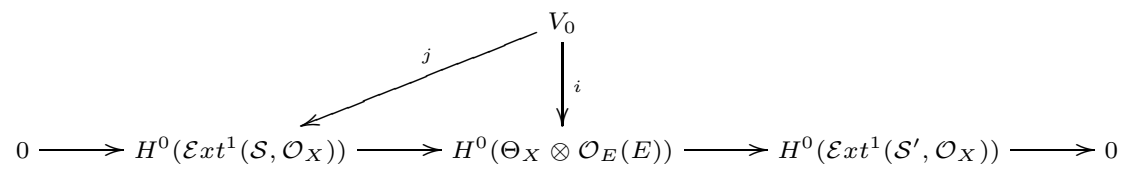


Corollary 2.4. Let $V_{1}$ be as in Proposition 2.2, the subspace of divisorial moduli of $X$. Then $\operatorname{dim} V_{1} \leq \frac{1}{2}\left(3 c_{2}-K^{2}\right)$.

Proof. From Proposition 2.2 we know that $\operatorname{dim} V_{1} \leq h^{0}\left(\mathcal{O}_{X}(F)\right)$. Thus we need to give an upper bound on the space of sections of the line bundle $\mathcal{O}_{X}(F)$. We may assume $F \neq 0$ (otherwise the assertion is obvious since the hypothesis $H^{1}\left(\Theta_{X}\right) \neq 0$ implies, by Yau's result, [21], and a theorem of Calabi-Vesentini,[4], that $\left.3 c_{2}-K^{2} \geq 4\right)$. Then we know that the linear system $|F|$ has at most finite number of base points. Blowing-up $X$ along the base locus of $|F|$ we obtain

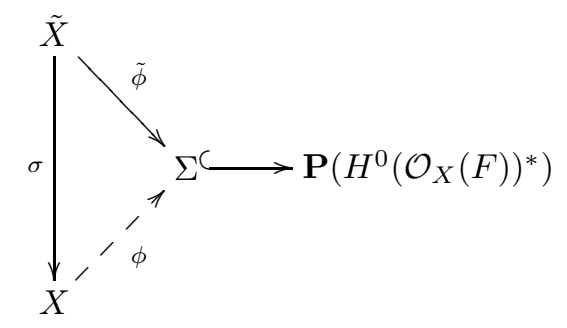

where $\Sigma$ is the image of the rational map $\phi$ defined by $\mathcal{O}_{X}(F)$ and $\sigma: \tilde{X} \longrightarrow X$ is a sequence of blowing-ups. We consider two cases according to the dimension of the image $\Sigma$.

1). $\operatorname{dim} \Sigma=2$. In this case we have the following estimate

$$
h^{0}\left(\mathcal{O}_{X}(F)\right) \leq \frac{1}{2} F^{2}+2 .
$$

Indeed, if $\Sigma$ is not a ruled surface then it is well-known that $h^{0}\left(\mathcal{O}_{X}(F)\right) \leq \frac{1}{2} \operatorname{deg} \Sigma+2$ (see e.g., Lemma 1.4,[2]). This combined with $\operatorname{deg} \Sigma \leq \frac{F^{2}}{\operatorname{deg} \phi}$ implies $h^{0}\left(\mathcal{O}_{X}(F)\right) \leq$ $\frac{1}{2} \frac{F^{2}}{\operatorname{deg} \phi}+2 \leq \frac{1}{2} F^{2}+2$.

If $\Sigma$ is ruled then we use $h^{0}\left(\mathcal{O}_{X}(F)\right) \leq \operatorname{deg} \Sigma+2 \leq \frac{F^{2}}{\operatorname{deg} \phi}+2$. Since the degree of $\phi$ must be at least 2 we obtain the inequality $(2.8)$ as well.

By Hodge Index $F^{2} \leq \frac{(F . K)^{2}}{K^{2}}$. Substituting this into (2.8) and using (1.13) together with Corollary 1.7 we obtain

$$
\operatorname{dim} V_{1} \leq h^{0}\left(\mathcal{O}_{X}(F)\right)<2(3 \alpha-1)\left(3 c_{2}-K^{2}\right)+2 \leq \frac{1}{4}\left(3 c_{2}-K^{2}\right)+2 .
$$

Since $3 c_{2}-K^{2}=4\left(9 \chi\left(\mathcal{O}_{X}\right)-K^{2}\right)$ is divisible by 4 it follows $\operatorname{dim} V_{1} \leq \frac{1}{4}\left(3 c_{2}-K^{2}\right)+1 \leq$ $\frac{1}{2}\left(3 c_{2}-K^{2}\right)$, where the last inequality follows from the assumption $H^{1}\left(\Theta_{X}\right) \neq 0$.

$2) \cdot \operatorname{dim} \Sigma=1$. The morphism $\tilde{\phi}: X \longrightarrow \Sigma$ factors through the normalization $\Sigma^{\prime}$ of $\Sigma$. Taking the Stein factorization we arrive to the following diagram

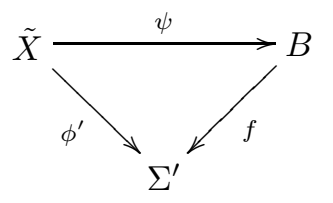

where $f$ is finite and $\psi$ is a surjective morphism with connected fibres. In particular, the strict transform $\tilde{F}$ of $F$ has the form $\phi^{\prime *}(D)$ for a divisor $D$ on $\Sigma^{\prime}$ with 
$\operatorname{deg}(D)=\operatorname{deg}(\Sigma)$. Putting $\tilde{C}$ to be the class of a smooth fibre of $\psi$ we obtain $\tilde{F}=\operatorname{deg}(\Sigma) \operatorname{deg}(f) \tilde{C}$. This implies that $F \cdot K=\tilde{F} \cdot \sigma^{*} K=\operatorname{deg}(\Sigma) \operatorname{deg}(f) C . K$, where $C=\sigma_{*}(\tilde{C})$. From this it follows

$$
\operatorname{dim} V_{1} \leq h^{0}\left(\mathcal{O}_{X}(F)\right) \leq \operatorname{deg}(\Sigma)+1=\frac{F . K}{\operatorname{deg}(f) C . K}+1 .
$$

Combining this with (1.13) and Corollary 1.7 we obtain

$$
\operatorname{dim} V_{1}<\frac{2\left(3 c_{2}-K^{2}\right)}{\operatorname{deg}(f) C . K}+1 \leq \frac{2\left(3 c_{2}-K^{2}\right)}{C . K}+1 .
$$

The asserted inequality follows from the following.

Claim. C. $K \geq 4$.

Proof of the Claim. The hypothesis $\alpha \leq \frac{3}{8}$ is equivalent to $K^{2} \geq \frac{96}{11} \chi\left(\mathcal{O}_{X}\right)$. In particular, $K^{2} \geq 9$ with equality holding if $\chi\left(\mathcal{O}_{X}\right)=1$. By Yau's theorem ([21]) such a surface must be a compact quotient of a unit ball in $\mathbf{C}^{2}$. By a result of CalabiVesentini ([4]) such surfaces are infinitesimally rigid which contradicts our assumption $H^{1}\left(\Theta_{X}\right) \neq 0$. So $K^{2} \geq 10$.

Assume $C . K \leq 3$. The Hodge Index and the inequality $K^{2} \geq 10$ imply $C^{2} \leq 0$. Since $C$ is nef divisor it follows that $C^{2}=0$ and $C . K=2$. Furthermore, $C^{2}=0$ implies that the linear system $|F|$ is base point free. So $X=\tilde{X} \longrightarrow B$ in (2.9) is a genus 2 fibration. However, by a result of Xiao (see [18]) such surfaces are subject to $\alpha \geq \frac{1}{2}$. Hence $C . K \geq 4$.

3. A study of locally supported moduli. Let $V_{0}$ be as in Proposition 2.2 . Our study of this subspace of $H^{1}\left(\Theta_{X}\right)$ goes via considerations of the extension $\mathcal{T}_{V_{0}}$. In particular, we go back to the diagram $(2.2)$. The divisor $E=c_{1}(\mathcal{S})$ has a stratification according to the rank of the morphism $\mu: \mathcal{G} \longrightarrow \Omega_{X}$ in (2.2). Let $\Gamma$ be the component of $E$ where $\mu$ vanishes. Then we can decompose the morphism $\mu$ as follows

$$
\mathcal{G} \stackrel{\mu_{1}}{\longrightarrow} \Omega_{X}(-\Gamma) \stackrel{\gamma}{\longrightarrow} \Omega_{X}
$$

where $\mu_{1}$ is a morphism which vanishes at most in codimension 2 and $\Gamma=(\gamma=0)$. This factorization yields the the following diagram

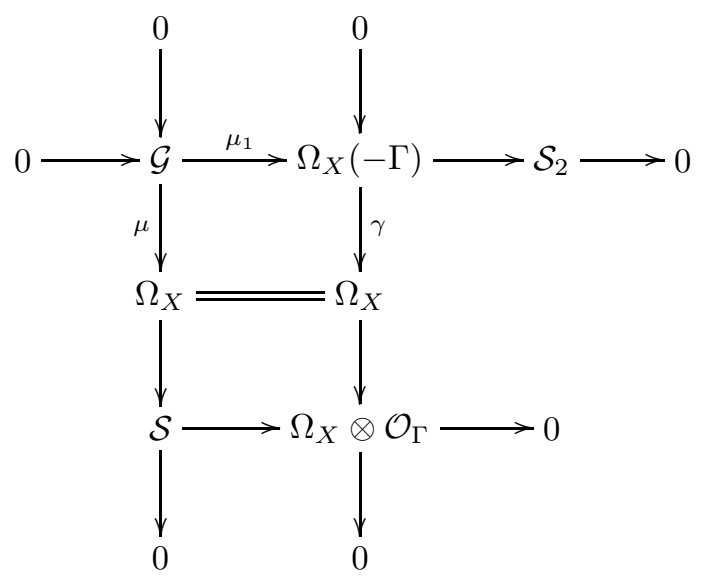


where $\mathcal{S}_{2}=\operatorname{coker}\left(\mu_{1}\right)$. From this diagram we deduce

$$
0 \longrightarrow \mathcal{S}_{2} \longrightarrow \mathcal{S} \longrightarrow \Omega_{X} \otimes \mathcal{O}_{\Gamma} \longrightarrow 0 .
$$

This implies

$$
E=c_{1}(\mathcal{S})=c_{1}\left(\mathcal{S}_{2}\right)+2 \Gamma \text {. }
$$

Dualizing (3.2) we obtain

$$
0 \longrightarrow \Theta_{X} \otimes \mathcal{O}_{\Gamma}(\Gamma) \longrightarrow \mathcal{E} x t^{1}\left(\mathcal{S}, \mathcal{O}_{X}\right) \longrightarrow \mathcal{E} x t^{1}\left(\mathcal{S}_{2}, \mathcal{O}_{X}\right) \longrightarrow 0 .
$$

This together with (2.7) imply

$$
0 \longrightarrow H^{0}\left(\Theta_{X} \otimes \mathcal{O}_{\Gamma}(\Gamma)\right) \longrightarrow H^{0}\left(\mathcal{E} x t^{1}\left(\mathcal{S}, \mathcal{O}_{X}\right)\right) \longrightarrow H^{0}\left(\mathcal{E} x t^{1}\left(\mathcal{S}_{2}, \mathcal{O}_{X}\right)\right) \text {. }
$$

Let $V_{0}^{\prime \prime}=V_{0} \cap H^{0}\left(\Theta_{X} \otimes \mathcal{O}_{\Gamma}(\Gamma)\right)$ and let $V_{0}^{\prime}$ be the image of $V_{0}$ in $H^{0}\left(\mathcal{E} x t^{1}\left(\mathcal{S}_{2}, \mathcal{O}_{X}\right)\right)$. Then we have

$$
\operatorname{dim} V_{0}=\operatorname{dim} V_{0}^{\prime \prime}+\operatorname{dim} V_{0}^{\prime} \leq h^{0}\left(\Theta_{X} \otimes \mathcal{O}_{\Gamma}(\Gamma)\right)+\operatorname{dim} V_{0}^{\prime} .
$$

We will now investigate the spaces $V_{0}^{\prime}$ and $V_{0}^{\prime \prime}$. An understanding of the latter one goes via the study of the divisor $\Gamma$. We begin by observing the following.

Lemma 3.1. The sheaf $\mathcal{O}_{\Gamma}(-L)$ is generated by global sections outside of a subscheme of dimension 0.

Proof. Restricting the diagram (2.2) to $\Gamma$ we obtain

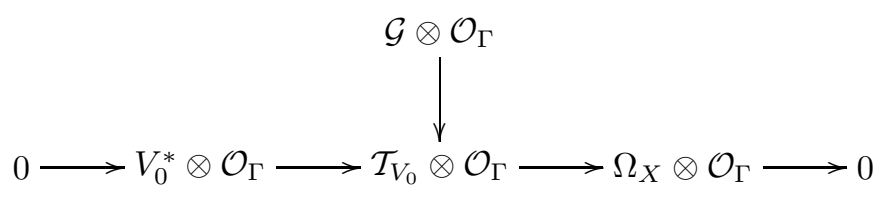

where the morphism $\mathcal{G} \otimes \mathcal{O}_{\Gamma} \longrightarrow \mathcal{T}_{V_{0}} \otimes \mathcal{O}_{\Gamma}$ factors through $V_{0}^{*} \otimes \mathcal{O}_{\Gamma}$. This gives a monomorphism $\mathcal{G} \otimes \mathcal{O}_{\Gamma} \longrightarrow V_{0}^{*} \otimes \mathcal{O}_{\Gamma}$. Taking determinant and dualizing yield the assertion.

To understand further properties of $\Gamma$ we let $\Gamma=\sum m_{C} C$ be the decomposition into distinct irreducible components. Put $\Lambda_{\Gamma}$ to be the sublattice of $N S(X)$ generated by the irreducible components $C$ 's.

Lemma 3.2. The intersection pairing restricted to $\Lambda_{\Gamma}$ is negative definite.

Proof. Let $L=L^{-}+L^{+}$be the Zariski decomposition of $L$ with $L^{+}$(resp. $L^{-}$) its positive (resp. negative) part. By Corollary 1.7 the positive part $L^{+} \neq 0$. From Lemma 3.1 it follows $C . L \leq 0$ for every irreducible component $C$ of $\Gamma$. This implies that either $C$ is in the support of $L^{-}$and then $L^{+} . C=0$, or $C$ is not in the support of 
$L^{-}$and then $C . L^{ \pm}=0$. Thus, we have $L^{+} . C=0$ for every irreducible component of $\Gamma$. This implies that the sublattice $\Lambda_{\Gamma}$ is orthogonal to $L^{+}$. By Hodge Index theorem, the intersection pairing is negative definite on $\Lambda_{\Gamma}$.

This lemma will enable us to show that the contribution of $V_{0}^{\prime \prime}$ amounts to counting certain rational curves in $\Gamma$.

Lemma 3.3. Assume $H^{0}\left(\Theta_{X} \otimes \mathcal{O}_{\Gamma}(\Gamma)\right) \neq 0$. Then the following holds.

a) There exists a decreasing sequence of components of $\Gamma$

$$
\Gamma \supset \Gamma_{0} \supset \Gamma_{1} \supset \cdots \supset \Gamma_{N-1} \supset \Gamma_{N}
$$

such that $h^{0}\left(\Theta_{X} \otimes \mathcal{O}_{\Gamma}(\Gamma)\right)=h^{0}\left(\Theta_{X} \otimes \mathcal{O}_{\Gamma_{0}}\left(\Gamma_{0}\right)\right)>h^{0}\left(\Theta_{X} \otimes \mathcal{O}_{\Gamma_{1}}\left(\Gamma_{1}\right)\right)>\cdots>$ $h^{0}\left(\Theta_{X} \otimes \mathcal{O}_{\Gamma_{N-1}}\left(\Gamma_{N-1}\right)\right)>h^{0}\left(\Theta_{X} \otimes \mathcal{O}_{\Gamma_{N}}\left(\Gamma_{N}\right)\right)=0$.

b) For each $i \in\{0, \ldots, N-1\}$ there exists a rational curve $C_{i+1} \subset \Gamma_{i}-\Gamma_{i+1}$ such that $C_{i+1} \cdot \Gamma_{i}=-1$ or -2 .

c) $h^{0}\left(\Theta_{X} \otimes \mathcal{O}_{\Gamma_{i}}\left(\Gamma_{i}\right)\right)-h^{0}\left(\Theta_{X} \otimes \mathcal{O}_{\Gamma_{i+1}}\left(\Gamma_{i+1}\right)\right) \leq 3+C_{i+1} . \Gamma_{i}$ for every $i \in\{0, \ldots, N-1\}$. In particular,

$$
h^{0}\left(\Theta_{X} \otimes \mathcal{O}_{\Gamma}(\Gamma)\right) \leq 3 N+\sum_{i=0}^{N-1} C_{i+1} \cdot \Gamma_{i} \leq 2 N
$$

Proof. Let $\Gamma_{0}$ be a smallest component of $\Gamma$ with the property $H^{0}\left(\Theta_{X} \otimes \mathcal{O}_{\Gamma}(\Gamma)\right)=$ $H^{0}\left(\Theta_{X} \otimes \mathcal{O}_{\Gamma_{0}}\left(\Gamma_{0}\right)\right)$. Choose a reduced irreducible component $C_{1}$ of $\Gamma_{0}$ such that

$$
C_{1} \cdot \Gamma_{0}=\min \left\{C \cdot \Gamma_{0} \mid C \text { is an irreducible component of } \Gamma_{0}\right\} .
$$

From Lemma 3.2 it follows $C_{1} \cdot \Gamma_{0}<0$. The definition of $\Gamma_{0}$ implies that the restriction morphism $H^{0}\left(\Theta_{X} \otimes \mathcal{O}_{\Gamma_{0}}\left(\Gamma_{0}\right)\right) \longrightarrow H^{0}\left(\Theta_{X} \otimes \mathcal{O}_{C_{1}}\left(\Gamma_{0}\right)\right)$ is nonzero. Consider the normal sequence of $C_{1}$ tensored with $\mathcal{O}_{C_{1}}\left(\Gamma_{0}\right)$

$$
0 \longrightarrow \Theta_{C_{1}} \otimes \mathcal{O}_{C_{1}}\left(\Gamma_{0}\right) \longrightarrow \Theta_{X} \otimes \mathcal{O}_{C_{1}}\left(\Gamma_{0}\right) \longrightarrow \mathcal{O}_{C_{1}}\left(C_{1}+\Gamma_{0}\right) .
$$

Since $H^{0}\left(\mathcal{O}_{C_{1}}\left(C_{1}+\Gamma_{0}\right)\right)=0$ it follows $H^{0}\left(\Theta_{C_{1}} \otimes \mathcal{O}_{C_{1}}\left(\Gamma_{0}\right)\right)=H^{0}\left(\Theta_{X} \otimes \mathcal{O}_{C_{1}}\left(\Gamma_{0}\right)\right) \neq 0$. This and $C_{1} \cdot \Gamma_{0}<0$ imply that $C_{1}$ is rational and $C_{1} \cdot \Gamma_{0}=-1$ or -2 . Set $\Gamma_{0}^{\prime}=\Gamma_{0}-C_{1}$. From the exact sequence

$$
0 \longrightarrow \Theta_{X} \otimes \mathcal{O}_{\Gamma_{0}^{\prime}}\left(\Gamma_{0}^{\prime}\right) \longrightarrow \Theta_{X} \otimes \mathcal{O}_{\Gamma_{0}}\left(\Gamma_{0}\right) \longrightarrow \Theta_{X} \otimes \mathcal{O}_{C_{1}}\left(\Gamma_{0}\right) \longrightarrow 0
$$

we deduce $h^{0}\left(\Theta_{X} \otimes \mathcal{O}_{\Gamma_{0}}\left(\Gamma_{0}\right)\right)-h^{0}\left(\Theta_{X} \otimes \mathcal{O}_{\Gamma_{0}^{\prime}}\left(\Gamma_{0}^{\prime}\right)\right) \leq h^{0}\left(\Theta_{X} \otimes \mathcal{O}_{C_{1}}\left(\Gamma_{0}\right)\right) \leq 3+C_{1} \cdot \Gamma_{0}$. If $h^{0}\left(\Theta_{X} \otimes \mathcal{O}_{\Gamma_{0}^{\prime}}\left(\Gamma_{0}^{\prime}\right)\right)=0$, then we set $\Gamma_{1}=\Gamma_{0}^{\prime}$ and the sequence $\Gamma_{0} \supset \Gamma_{1}$ has the required properties. If $h^{0}\left(\Theta_{X} \otimes \mathcal{O}_{\Gamma_{0}^{\prime}}\left(\Gamma_{0}^{\prime}\right)\right) \neq 0$, then we repeat the above argument with $\Gamma_{0}^{\prime}$ in place of $\Gamma$. This will define $\Gamma_{1}$ and $C_{2}$. The process will terminate after a finite number of steps yielding a decreasing sequence $\Gamma \supset \Gamma_{0} \supset \Gamma_{1} \supset \cdots \supset \Gamma_{N-1} \supset \Gamma_{N}$ with the asserted properties. In particular, summing up all the inequalities in c) of the lemma we obtain

$$
h^{0}\left(\Theta_{X} \otimes \mathcal{O}_{\Gamma}(\Gamma)\right) \leq 3 N+\sum_{i=0}^{N-1} C_{i+1} \cdot \Gamma_{i} \leq 2 N
$$


REMARK 3.4. Dualizing the bottom sequence in (2.2) we obtain the morphism

$$
V_{0} \otimes \mathcal{O}_{X} \longrightarrow \mathcal{E} x t^{1}\left(\mathcal{S}, \mathcal{O}_{X}\right)
$$

which is surjective outside a subscheme of dimension 0. This combined with the proof of Lemma 3.3 implies that $c_{1}\left(\mathcal{S}_{2}\right)$ in (3.3) is nonzero and the morphism $V_{0} \otimes \mathcal{O}_{X} \longrightarrow$ $\mathcal{E} x t^{1}\left(\mathcal{S}_{2}, \mathcal{O}_{X}\right)$ is surjective outside a subscheme of dimension 0 . Hence, the space $V_{0}^{\prime}$ in (3.5) is nonzero.

Next we turn to a study of $V_{0}^{\prime}$. From the short exact sequence on the top of (3.1) it follows that $V_{0}^{\prime}$ injects into the kernel of $H^{1}\left(\Theta_{X}(\Gamma)\right) \longrightarrow H^{1}\left(\mathcal{G}^{*}\right)$. This implies that the morphism $\mu_{1}$ in (3.1) lifts to the extension of $\Omega_{X}(-\Gamma)$ corresponding to $V_{0}^{\prime}$ (viewed as a subspace of $H^{1}\left(\Theta_{X}(\Gamma)\right)$ ), i.e. we have the following diagram

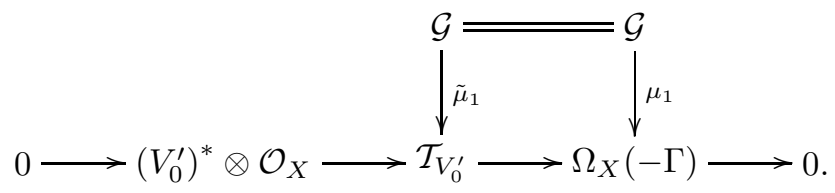

Factoring out by the torsion of the quotient $\mathcal{T}_{V_{0}^{\prime}} / \mathcal{G}$ we obtain the diagram

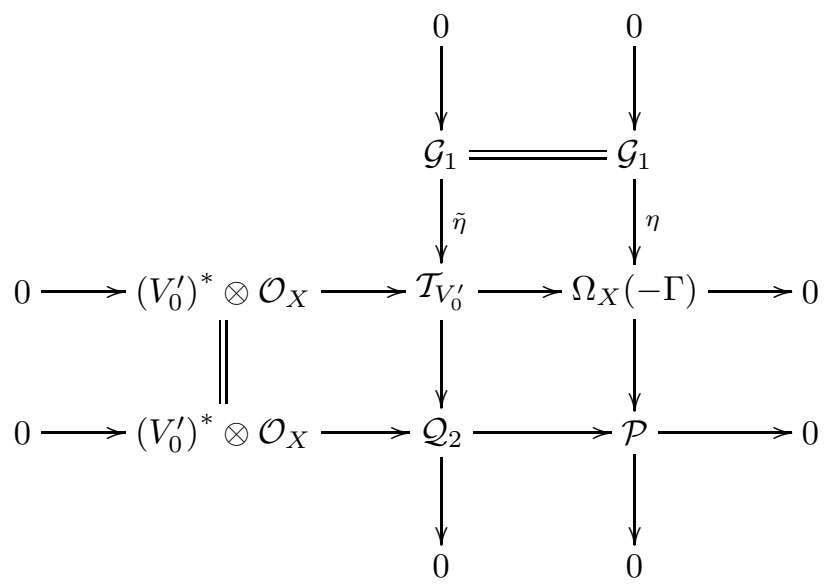

analogous to the one in (2.2). As before the morphism $\eta: \mathcal{G}_{1} \longrightarrow \Omega_{X}(-\Gamma)$ is generically an isomorphism. But this time it vanishes at most in codimension 2. This implies that the sheaf $\mathcal{P}$ has rank 1 outside of a subscheme of dimension 0 on the support of $\mathcal{P}$. Set $E_{2}=c_{1}(\mathcal{P})$ and $L_{1}^{\prime}=c_{1}\left(\mathcal{G}_{1}\right)$. Since the extension $\mathcal{T}_{V_{0}^{\prime}}$ is nontrivial it follows that $E_{2}$ is an effective nonzero divisor. By definition of $\mathcal{G}_{1}$ its first Chern class $L_{1}^{\prime}=c_{1}(\mathcal{G})+c_{1}\left(\operatorname{Tor}\left(\mathcal{T}_{V_{0}^{\prime}} / \mathcal{G}\right)\right)=L+c_{1}\left(\operatorname{Tor}\left(\mathcal{T}_{V_{0}^{\prime}} / \mathcal{G}\right)\right)$. We have seen that $L$ is a divisor of D-dimension 2. So the same holds for $L_{1}^{\prime}$. Furthermore, dualizing the bottom sequence in (3.6) we obtain

$$
\operatorname{dim} V_{0}^{\prime} \leq h^{0}\left(\mathcal{E} x t^{1}\left(\mathcal{P}, \mathcal{O}_{X}\right)\right)
$$

To obtain the upper bound on the dimension of $V_{0}^{\prime}$ we compare the extension $\mathcal{T}_{V_{0}^{\prime}}$ with a one-dimensional extension.

Fix a nonzero vector $\xi$ in $V_{0}^{\prime}$. Viewing it as a cohomology class in $H^{1}\left(\Theta_{X}(\Gamma)\right)$ 
(recall: we identify $V_{0}^{\prime}$ with its image in $H^{1}\left(\Theta_{X}(\Gamma)\right)$ ) we consider the extension corresponding to $\xi$

$$
0 \longrightarrow \mathcal{O}_{X} \longrightarrow \mathcal{T}_{\xi} \longrightarrow \Omega_{X}(-\Gamma) \longrightarrow 0
$$

This extension and $\mathcal{T}_{V_{0}^{\prime}}$ are related by the following diagram

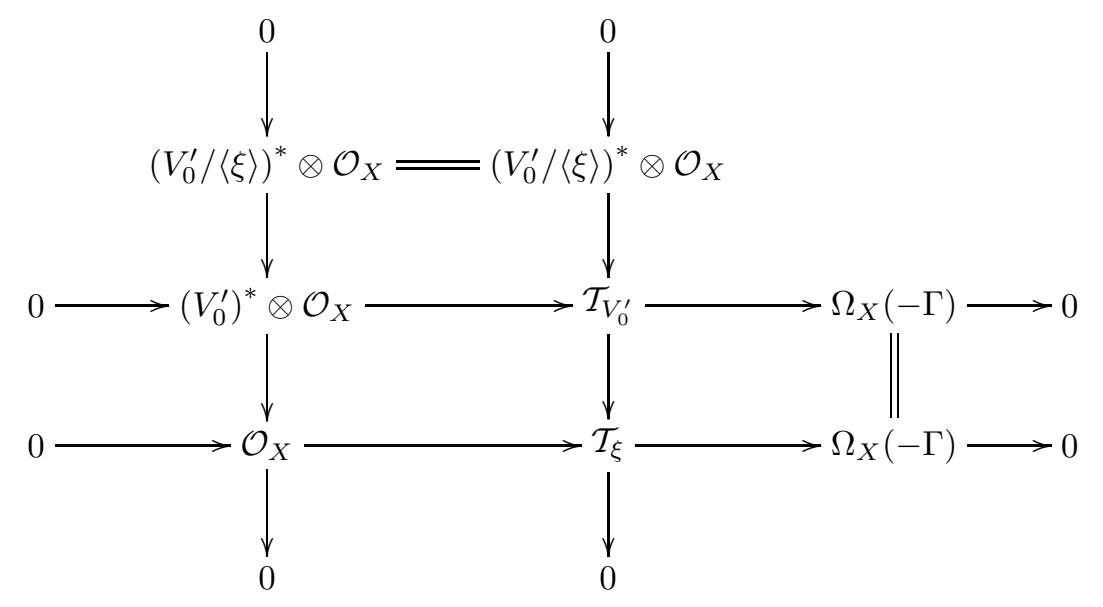

where $\langle\xi\rangle$ is the one-dimensional subspace of $H^{1}\left(\Theta_{X}(\Gamma)\right)$ spanned by $\xi$. Combining this with the middle column in (3.6) we obtain

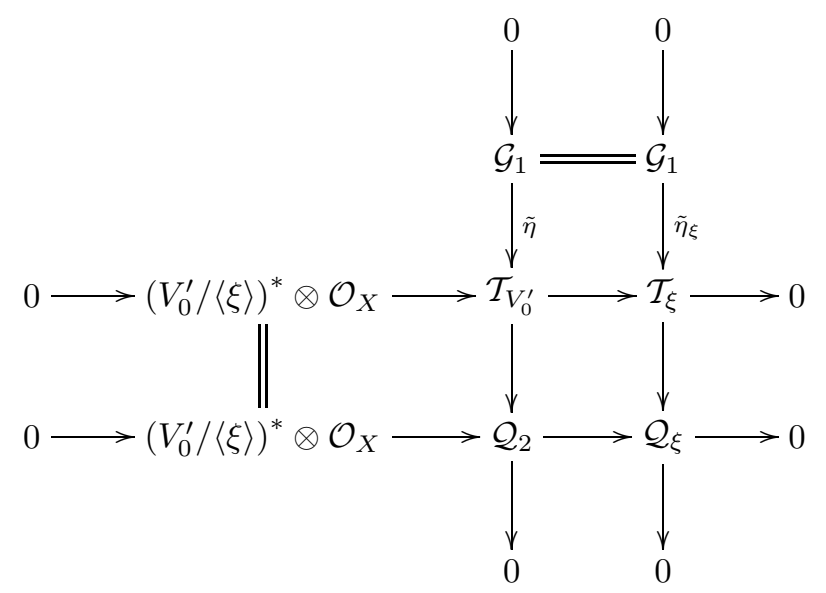

where $\mathcal{Q}_{\xi}$ is the cokernel of $\tilde{\eta}_{\xi}$.

Lemma 3.5. For a general $\xi$ in $V_{0}^{\prime}$ the sheaf $\mathcal{Q}_{\xi}$ is torsion-free.

Proof. It is enough to show that the morphism $\tilde{\eta}_{\xi}$ in (3.9) drops its rank at most at finite set of points. Observe that $\tilde{\eta}_{\xi}$ drops its rank where

(i) $\tilde{\eta}$ drops its rank and where

(ii) $\operatorname{Im}(\tilde{\eta})$ intersects nontrivially the subbundle $\left(V_{0}^{\prime} /\langle\xi\rangle\right)^{*} \otimes \mathcal{O}_{X}$.

The set of points in (i) is finite since $\mathcal{Q}_{2}=\operatorname{coker}(\tilde{\eta})$ is torsion-free. Turning to 
the points in (ii) we use the fact that $\left.\eta\right|_{E_{2}}: \mathcal{G}_{1} \otimes \mathcal{O}_{E_{2}} \longrightarrow \Omega_{X}(-\Gamma) \otimes \mathcal{O}_{E_{2}}$ drops its rank precisely by 1 outside of a finite set of points. Then the kernel of $\left.\eta\right|_{E_{2}}$ gives rise to a one-dimensional subscheme in $\mathbf{P}\left(V_{0}^{\prime *}\right)$ and the points in (ii) are the points of the intersection of the hyperplane $\mathbf{P}\left(\left(V_{0}^{\prime} /\langle\xi\rangle\right)^{*}\right)$ with this one dimensional subscheme. It is clear that this intersection is finite for a general choice of $\xi$. $\square$

We are now in the position to give an upper bound on the dimension of $V_{0}^{\prime}$. From Lemma 3.5 it follows that $\mathcal{Q}_{\xi}$ has the form $\mathcal{I}_{Z_{\xi}}\left(E_{2}\right)$, where $\mathcal{I}_{Z_{\xi}}$ is the sheaf of ideals of some 0-dimensional subscheme $Z_{\xi}$. The right-hand column in (3.9) combined with the defining sequence of $\mathcal{T}_{\xi}$ gives the following diagram

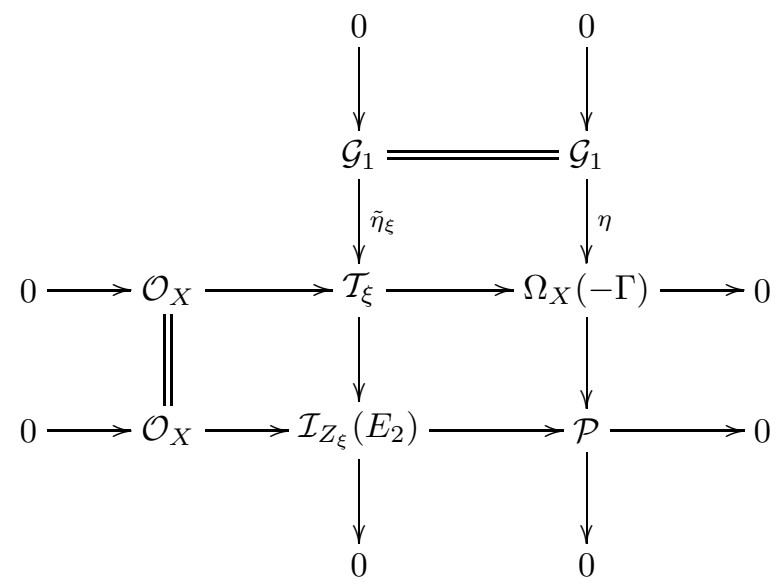

Dualizing the bottom sequence we have

$$
0 \longrightarrow \mathcal{O}_{X}\left(-E_{2}\right) \longrightarrow \mathcal{O}_{X} \longrightarrow \mathcal{E} x t^{1}\left(\mathcal{P}, \mathcal{O}_{X}\right) \longrightarrow \mathcal{E} x t^{1}\left(\mathcal{I}_{Z_{\xi}}\left(E_{2}\right), \mathcal{O}_{X}\right) \longrightarrow 0
$$

This implies $h^{0}\left(\mathcal{E} x t^{1}\left(\mathcal{P}, \mathcal{O}_{X}\right)\right) \leq h^{0}\left(\mathcal{O}_{E_{2}}\right)+h^{0}\left(\mathcal{E} x t^{1}\left(\mathcal{I}_{Z_{\xi}}\left(E_{2}\right), \mathcal{O}_{X}\right)\right)=h^{0}\left(\mathcal{O}_{E_{2}}\right)+$ $\chi\left(\mathcal{E} x t^{1}\left(\mathcal{I}_{Z_{\xi}}\left(E_{2}\right), \mathcal{O}_{X}\right)\right)$. Substituting into (3.7) we obtain

$$
\operatorname{dim} V_{0}^{\prime} \leq h^{0}\left(\mathcal{O}_{E_{2}}\right)+\chi\left(\mathcal{E} x t^{1}\left(\mathcal{I}_{Z_{\xi}}\left(E_{2}\right), \mathcal{O}_{X}\right)\right) .
$$

Proposition 3.6. Let $E_{2}^{\prime}=E_{2}+2 \Gamma$. Then the following inequality holds:

$$
\operatorname{dim} V_{0}^{\prime} \leq \frac{1}{3}\left(3 c_{2}-K^{2}\right)+\frac{1}{6}\left(K \cdot E_{2}^{\prime}+\left(E_{2}^{\prime}\right)^{2}\right)-\Gamma^{2} .
$$

Proof. The asserted inequality is obtained by bounding two terms in (3.11). We begin by estimating the second term. Dualizing the column in the middle of (3.10) we obtain

$$
\chi\left(\mathcal{E} x t^{1}\left(\mathcal{I}_{Z_{\xi}}\left(E_{2}\right), \mathcal{O}_{X}\right)\right)=\chi\left(\mathcal{G}_{1}^{*}\right)-\chi\left(\mathcal{T}_{\xi}^{*}\right)+\chi\left(\mathcal{O}_{X}\left(-E_{2}\right)\right) .
$$

Applying the Riemann-Roch to the right-hand side yields

$$
\begin{aligned}
\chi\left(\mathcal{E} x t^{1}\left(\mathcal{I}_{Z_{\xi}}\left(E_{2}\right), \mathcal{O}_{X}\right)\right) & =\frac{1}{2}\left(L_{1}^{\prime}\right)^{2}-c_{2}\left(\mathcal{G}_{1}\right)+\frac{1}{2} E_{2}^{2}-\frac{1}{2}(K-2 \Gamma)^{2}+\left(c_{2}-K . \Gamma+\Gamma^{2}\right) \\
& =\frac{1}{2}\left(L_{1}^{\prime}\right)^{2}-c_{2}\left(\mathcal{G}_{1}\right)+\frac{1}{2} E_{2}^{2}-\frac{1}{2} K^{2}+c_{2}+K . \Gamma-\Gamma^{2} .
\end{aligned}
$$


By Miyaoka $c_{2}\left(\mathcal{G}_{1}\right) \geq \frac{1}{3}\left(L_{1}^{\prime}\right)^{2}$ (Remark 4.18,[12]). Substituting in (3.12) we obtain

$$
\begin{aligned}
\chi\left(\mathcal{E} x t^{1}\left(\mathcal{I}_{Z_{\xi}}\left(E_{2}\right), \mathcal{O}_{X}\right)\right) & \leq \frac{1}{6}\left(L_{1}^{\prime}\right)^{2}+\frac{1}{2} E_{2}^{2}-\frac{1}{2} K^{2}+c_{2}+K . \Gamma-\Gamma^{2} \\
& =\frac{1}{6}\left(K-2 \Gamma-E_{2}\right)^{2}+\frac{1}{2} E_{2}^{2}-\frac{1}{2} K^{2}+c_{2}+K . \Gamma-\Gamma^{2} \\
& =\frac{1}{3}\left(3 c_{2}-K^{2}\right)-\frac{1}{3} K \cdot E_{2}^{\prime}+\frac{1}{6}\left(E_{2}^{\prime}\right)^{2}+\frac{1}{2} E_{2}^{2}+K . \Gamma-\Gamma^{2}
\end{aligned}
$$

where $E_{2}^{\prime}=E_{2}+2 \Gamma$. This together with (3.11) imply

$$
\operatorname{dim} V_{0}^{\prime} \leq h^{0}\left(\mathcal{O}_{E_{2}}\right)+\frac{1}{3}\left(3 c_{2}-K^{2}\right)-\frac{1}{3} K \cdot E_{2}^{\prime}+\frac{1}{6}\left(E_{2}^{\prime}\right)^{2}+\frac{1}{2} E_{2}^{2}+K . \Gamma-\Gamma^{2} .
$$

To bound the first term in in the above inequality we use the following.

Lemma 3.7. Let $X$ be a smooth minimal surface of general type. Assume $K_{X}=$ $D+D^{\prime}$, where $D$ is effective and $D^{\prime}$ has the Zariski decomposition whose positive part is nonzero. Then $h^{0}\left(\mathcal{O}_{D}\right) \leq \frac{K . D-D^{2}}{2}$.

Let us assume this result and complete the proof of the proposition.

From the middle column of (3.10) it follows $K-2 \Gamma=L_{1}^{\prime}+E_{2}$. This gives the decomposition $K=E_{2}+\left(L_{1}^{\prime}+2 \Gamma\right)$ which satisfies the hypothesis of Lemma 3.7. So we deduce $h^{0}\left(\mathcal{O}_{E_{2}}\right) \leq \frac{1}{2}\left(K . E_{2}-E_{2}^{2}\right)$. Substituting in (3.13) gives

$$
\begin{aligned}
\operatorname{dim} V_{0}^{\prime} & \leq \frac{1}{3}\left(3 c_{2}-K^{2}\right)-\frac{1}{3} K \cdot E_{2}^{\prime}+\frac{1}{2} K \cdot E_{2}+K \cdot \Gamma+\frac{1}{6}\left(E_{2}^{\prime}\right)^{2}-\Gamma^{2} \\
& =\frac{1}{3}\left(3 c_{2}-K^{2}\right)+\frac{1}{6} K \cdot E_{2}^{\prime}+\frac{1}{6}\left(E_{2}^{\prime}\right)^{2}-\Gamma^{2}
\end{aligned}
$$

which is the inequality asserted in the proposition. We turn now to a proof of Lemma 3.7.

Proof of Lemma 3.7. We may assume $D \neq 0$ (otherwise there is nothing to prove). If $D$ is nef and big then by Ramanujam's vanishing theorem (Theorem 8.1, IV, [1]) $h^{0}\left(\mathcal{O}_{D}\right)=1$. On the other hand $K . D-D^{2}=D . D^{\prime}$. Writing $D^{\prime}=P^{\prime}+N^{\prime}$, the Zariski decomposition of $D^{\prime}$, where $P^{\prime}$ (resp. $N^{\prime}$ ) is its positive (resp. negative) part, we have $D . P^{\prime}>0$ (since $P^{\prime} \neq 0$ and $D$ is nef and big). So we have $D . D^{\prime}>0$. Since $K . D-D^{2}=D \cdot D^{\prime}$ is even we obtain $\frac{K . D-D^{2}}{2} \geq 1=h^{0}\left(\mathcal{O}_{D}\right)$.

If $D$ is nef and $D^{2}=0$, then one has the following decomposition $D=\sum_{i=1}^{N} D_{i}$ where $D_{i} . D_{j}=0$, for every $i, j$ and $h^{0}\left(\mathcal{O}_{D_{i}}\right)=1$, for every $i$. In particular,

$$
h^{0}\left(\mathcal{O}_{D}\right) \leq N \leq \frac{1}{2} \sum_{i=1}^{N} D_{i} \cdot K=\frac{1}{2} K . D .
$$

The general case can be reduced to the case of a nef divisor as follows. By RiemannRoch for $\mathcal{O}_{D}$ we have :

$$
h^{0}\left(\mathcal{O}_{D}\right)-h^{1}\left(\mathcal{O}_{D}\right)=-\frac{K \cdot D+D^{2}}{2} .
$$

By Serre duality $h^{1}\left(\mathcal{O}_{D}\right)=h^{0}\left(\mathcal{O}_{D}\left(D+K_{X}\right)\right)$. Set $D=D_{0}$ and let $C_{0}$ be a reduced, irreducible component of $D_{0}$ for which $D_{0} \cdot C_{0}<0$. Put $D_{1}=D_{0}-C_{0}$ and consider the exact sequence

$$
0 \longrightarrow \mathcal{O}_{D_{1}}\left(D_{1}+K_{X}\right) \longrightarrow \mathcal{O}_{D_{0}}\left(D_{0}+K_{X}\right) \longrightarrow \mathcal{O}_{C_{0}}\left(D_{0}+K_{X}\right) \longrightarrow 0 .
$$


From the cohomology sequence we deduce

$$
h^{0}\left(\mathcal{O}_{D_{0}}\left(D_{0}+K_{X}\right)\right)-h^{0}\left(\mathcal{O}_{D_{1}}\left(D_{1}+K_{X}\right)\right) \leq h^{0}\left(\mathcal{O}_{C_{0}}\left(D_{0}+K_{X}\right)\right) \leq K . C_{0} .
$$

Repeating the above procedure a finite number of times we obtain the decomposition $D=D_{n}+\sum_{i=0}^{n-1} C_{i}$, where $D_{n}$ is a nef divisor. Summing up the inequalities of type (3.15) we obtain

$$
h^{0}\left(\mathcal{O}_{D}\left(D+K_{X}\right)\right)-h^{0}\left(\mathcal{O}_{D_{n}}\left(D_{n}+K_{X}\right)\right) \leq \sum_{i=0}^{n-1} K . C_{i} .
$$

From the Riemann-Roch applied to $D_{n}$ we have

$$
h^{0}\left(\mathcal{O}_{D_{n}}\left(D_{n}+K_{X}\right)\right)=h^{0}\left(\mathcal{O}_{D_{n}}\right)+\frac{K \cdot D_{n}+D_{n}{ }^{2}}{2}
$$

Substituting this in (3.16) we obtain

$$
h^{1}\left(\mathcal{O}_{D}\right)=h^{0}\left(\mathcal{O}_{D}\left(D+K_{X}\right)\right) \leq h^{0}\left(\mathcal{O}_{D_{n}}\right)+\frac{K . D_{n}+D_{n}{ }^{2}}{2}+\sum_{i=0}^{n-1} K . C_{i} .
$$

This and (3.14) imply

$$
\begin{aligned}
h^{0}\left(\mathcal{O}_{D}\right) & \leq h^{0}\left(\mathcal{O}_{D_{n}}\right)+\frac{K \cdot D_{n}+D_{n}{ }^{2}}{2}+\sum_{i=0}^{n-1} K \cdot C_{i}-\frac{K \cdot D+D^{2}}{2} \\
& =h^{0}\left(\mathcal{O}_{D_{n}}\right)+\frac{1}{2} \sum_{i=0}^{n-1} K \cdot C_{i}+\frac{D_{n}{ }^{2}-D^{2}}{2}
\end{aligned}
$$

If $D_{n}{ }^{2}=0$, then by the first part of the argument $h^{0}\left(\mathcal{O}_{D_{n}}\right) \leq \frac{1}{2} K \cdot D_{n}$. Substituting this in (3.17) we obtain the assertion.

If $D_{n}{ }^{2}>0$, then $h^{0}\left(\mathcal{O}_{D_{n}}\right)=1$ and (3.17) imply

$$
h^{0}\left(\mathcal{O}_{D}\right) \leq 1+\frac{1}{2} \sum_{i=0}^{n-1} K . C_{i}+\frac{D_{n}^{2}-D^{2}}{2} .
$$

Since $D_{n}{ }^{2} \leq D_{n} \cdot D=D_{n} \cdot\left(K-D^{\prime}\right)=D_{n} \cdot K-D_{n} \cdot D^{\prime}$ we obtain

$$
h^{0}\left(\mathcal{O}_{D}\right) \leq 1+\frac{1}{2}\left(K . D-D^{2}\right)-\frac{1}{2} D_{n} \cdot D^{\prime} .
$$

Using the Zariski decomposition of $D^{\prime}=P^{\prime}+N^{\prime}$ we have $D_{n} \cdot D^{\prime}=D_{n} \cdot P^{\prime}+D_{n} \cdot N^{\prime} \geq$ $D_{n} \cdot P^{\prime}>0$. So $D_{n} \cdot D^{\prime} \geq 1$ and we have

$$
h^{0}\left(\mathcal{O}_{D}\right) \leq \frac{1}{2}\left(K . D-D^{2}\right)+\frac{1}{2}
$$

Since $\frac{1}{2}\left(K . D-D^{2}\right)$ is an integer it follows $h^{0}\left(\mathcal{O}_{D}\right) \leq \frac{1}{2}\left(K . D-D^{2}\right)$.

Corollary 3.8. Let $V_{0}^{\prime}$ be as in Proposition 3.6 then

$$
\operatorname{dim} V_{0}^{\prime}< \begin{cases}2 \alpha\left(3 c_{2}-K^{2}\right)-\Gamma^{2}-\frac{1}{6}\left(1+2 \frac{K \cdot E_{2}^{\prime}}{K^{2}}+\frac{K . F}{K^{2}}\right) K . F, & \text { if } r_{1}=3 \\ 3 c_{2}-K^{2}-\Gamma^{2}, & \text { if } r_{1}=2\end{cases}
$$


where $F$ is as in Proposition 2.2, $\Gamma$ and $E_{2}^{\prime}$ are as in Proposition 3.6

Proof. If $r_{1}=3$, then from (1.13), (3.3) and the definition of $E_{2}^{\prime}$ in Proposition 3.6 it follows

$$
K \cdot E_{2}^{\prime} \leq K . E_{1}-K . F .
$$

This and the Hodge index imply

$$
\left(E_{2}^{\prime}\right)^{2} \leq \frac{\left(K \cdot E_{1}-K \cdot F\right)^{2}}{K^{2}}=\frac{\left(K \cdot E_{1}\right)^{2}}{K^{2}}-2 \frac{K \cdot E_{1}}{K^{2}} K \cdot F+\frac{(K \cdot F)^{2}}{K^{2}} .
$$

Substituting the two inequalities above in the inequality of Proposition 3.6 we obtain

$$
\begin{aligned}
\operatorname{dim} V_{0}^{\prime} & \leq \frac{1}{3}\left(3 c_{2}-K^{2}\right)+\frac{1}{6}\left(K \cdot E_{1}+\frac{\left(K \cdot E_{1}\right)^{2}}{K^{2}}\right)-\frac{1}{6}\left(K \cdot F+2 \frac{K \cdot E_{1}}{K^{2}} K \cdot F-\frac{(K \cdot F)^{2}}{K^{2}}\right)-\Gamma^{2} \\
& \leq \frac{1}{3}\left(3 c_{2}-K^{2}\right)+\frac{1}{6}\left(K \cdot E_{1}+\frac{\left(K \cdot E_{1}\right)^{2}}{K^{2}}\right)-\frac{1}{6}\left(1+2 \frac{K \cdot E_{2}^{\prime}}{K^{2}}+\frac{K \cdot F}{K^{2}}\right) K \cdot F-\Gamma^{2}(3.18)
\end{aligned}
$$

This and the bound on K.E $E_{1}$ in Corollary 1.7 imply

$$
\begin{aligned}
\operatorname{dim} V_{0}^{\prime} & <\frac{1}{3}\left(3 c_{2}-K^{2}\right)+\frac{1}{3}(1+2(3 \alpha-1))\left(3 c_{2}-K^{2}\right)-\frac{1}{6}\left(1+2 \frac{K \cdot E_{2}^{\prime}}{K^{2}}+\frac{K \cdot F}{K^{2}}\right) K \cdot F-\Gamma^{2} \\
& =2 \alpha\left(3 c_{2}-K^{2}\right)-\frac{1}{6}\left(1+2 \frac{K \cdot E_{2}^{\prime}}{K^{2}}+\frac{K \cdot F}{K^{2}}\right) K \cdot F-\Gamma^{2}
\end{aligned}
$$

If $r_{1}=2$, then we use the inequality (1.16) with $E_{1}$ replaced by $E_{2}^{\prime}$. This yields

$$
K . E_{2}^{\prime}+\left(E_{2}^{\prime}\right)^{2}<4\left(3 c_{2}-K^{2}\right) .
$$

Substituting into the inequality in Proposition 3.6 we obtain the assertion. $\square$

Corollary 3.9. Let $V_{0}$ be as in Proposition 2.2, the subspace of the locally supported moduli of $X$. Then

$$
\operatorname{dim} V_{0}< \begin{cases}2 \alpha\left(3 c_{2}-K^{2}\right)-\Gamma^{2}+2 N-\frac{1}{6}\left(1+2 \frac{K \cdot E_{2}^{\prime}}{K^{2}}+\frac{K \cdot F}{K^{2}}\right) K . F, & \text { if } r_{1}=3 \\ 3 c_{2}-K^{2}-\Gamma^{2}+2 N, & \text { if } r_{1}=2\end{cases}
$$

where $\Gamma$ and $N$ are as in Lemma 3.3, $F$ and $E_{2}^{\prime}$ are as in Corollary 3.8.

Proof. The inequalities of Corollary 3.8 and the last inequality in Lemma 3.3,c) substituted in (3.5) yield the assertion. $\square$

Combining the result of Corollary 3.9 with our analysis of the divisorial moduli of $X$ in the proof of Corollary 2.4 will yield a bound on the dimension of the space of infinitesimal deformations of $X$.

Corollary 3.10. Let $\alpha \leq \frac{3}{8}$ and $h^{1}\left(\Theta_{X}\right)>2$ and let $\Gamma$ and $N$ be as in Lemma 3.3. Then

$$
h^{1}\left(\Theta_{X}\right) \leq 3 c_{2}-K^{2}-\Gamma^{2}+2 N .
$$


Proof. If $r_{1}=3$, then combining Proposition 2.2 with Corollary 3.9 we obtain

$$
h^{1}\left(\Theta_{X}\right)<2 \alpha\left(3 c_{2}-K^{2}\right)-\Gamma^{2}+2 N-\frac{1}{6}\left(1+2 \frac{K . E_{2}^{\prime}}{K^{2}}+\frac{K . F}{K^{2}}\right) K . F+\operatorname{dim} V_{1} .
$$

If $F=0$, then $\operatorname{dim} V_{1}=1$ and we obtain

$$
h^{1}\left(\Theta_{X}\right)<2 \alpha\left(3 c_{2}-K^{2}\right)-\Gamma^{2}+2 N+1 .
$$

Combining this with $\alpha \leq \frac{3}{8}$ we obtain the asserted inequality.

If $F \neq 0$, we argue as in the proof of Corollary 2.4. plies

1). The linear system $|F|$ is not composed of a pencil. Then (2.8) im-

$$
\operatorname{dim} V_{1} \leq \frac{1}{2} F^{2}+2 \leq \frac{1}{2} \frac{(K \cdot F)^{2}}{K^{2}}+2 .
$$

Substituting the last expression above in (3.20) yields

$$
\begin{aligned}
h^{1}\left(\Theta_{X}\right) & <2 \alpha\left(3 c_{2}-K^{2}\right)-\Gamma^{2}+2 N-\frac{1}{6}\left(1+2 \frac{K \cdot E_{2}^{\prime}}{K^{2}}+\frac{K \cdot F}{K^{2}}\right) K \cdot F+\frac{1}{2} \frac{(K \cdot F)^{2}}{K^{2}}+2 \\
& =2 \alpha\left(3 c_{2}-K^{2}\right)-\Gamma^{2}+2 N+2+\frac{1}{6}\left(2 \frac{K \cdot F}{K^{2}}-1-2 \frac{K \cdot E_{2}^{\prime}}{K^{2}}\right) K \cdot F \\
& \leq 2 \alpha\left(3 c_{2}-K^{2}\right)-\Gamma^{2}+2 N+2+\frac{1}{6}\left(2 \frac{K \cdot E_{1}}{K^{2}}-1-4 \frac{K \cdot E_{2}^{\prime}}{K^{2}}\right) K . F .
\end{aligned}
$$

Using the upper bound for $\frac{K \cdot E_{1}}{K^{2}}$ from Corollary 1.7 we obtain

$$
h^{1}\left(\Theta_{X}\right)<2 \alpha\left(3 c_{2}-K^{2}\right)-\Gamma^{2}+2 N+2+\frac{1}{6}\left(12 \alpha-5-4 \frac{K \cdot E_{2}^{\prime}}{K^{2}}\right) K . F .
$$

This combined with $\alpha \leq \frac{3}{8}$ gives

$$
h^{1}\left(\Theta_{X}\right)<\frac{3}{4}\left(3 c_{2}-K^{2}\right)-\Gamma^{2}+2 N+2-\frac{1}{6}\left(\frac{1}{2}+4 \frac{K \cdot E_{2}^{\prime}}{K^{2}}\right) K . F
$$

which implies $h^{1}\left(\Theta_{X}\right) \leq 3 c_{2}-K^{2}-\Gamma^{2}+2 N$ as asserted.

2). The linear system $|F|$ is composed of a pencil. Then (2.10) together with Claim in the proof of Corollary 2.4 imply $\operatorname{dim} V_{1} \leq \frac{1}{4} K . F+1$. Substituting in (3.20) we obtain

$$
\begin{aligned}
h^{1}\left(\Theta_{X}\right) & <2 \alpha\left(3 c_{2}-K^{2}\right)-\Gamma^{2}+2 N+1+\frac{1}{12} K \cdot F-\left(2 \frac{K \cdot E_{2}^{\prime}}{K^{2}}+\frac{K \cdot F}{K^{2}}\right) K \cdot F \\
& =2 \alpha\left(3 c_{2}-K^{2}\right)-\Gamma^{2}+2 N+1+\frac{1}{12} K \cdot E_{1}-\frac{1}{12} K \cdot E_{2}^{\prime}-\left(2 \frac{K \cdot E_{2}^{\prime}}{K^{2}}+\frac{K \cdot F}{K^{2}}\right) K \cdot F \\
& <\left(2 \alpha+\frac{1}{6}\right)\left(3 c_{2}-K^{2}\right)-\Gamma^{2}+2 N+1-\frac{1}{12} K \cdot E_{2}^{\prime}-\left(2 \frac{K \cdot E_{2}^{\prime}}{K^{2}}+\frac{K \cdot F}{K^{2}}\right) K \cdot F \\
& \leq \frac{11}{12}\left(3 c_{2}-K^{2}\right)-\Gamma^{2}+2 N+1-\frac{1}{12} K \cdot E_{2}^{\prime}-\left(2 \frac{K \cdot E_{2}^{\prime}}{K^{2}}+\frac{K \cdot F}{K^{2}}\right) K \cdot F \cdot
\end{aligned}
$$


From this one immediately deduces the inequality $h^{1}\left(\Theta_{X}\right) \leq 3 c_{2}-K^{2}-\Gamma^{2}+2 N$.

If $r_{1}=2$, then $X$ has locally supported moduli only, i.e. $H^{1}\left(\Theta_{X}\right)=V_{0}$. This together with the second inequality of Corollary 3.9 imply $h^{1}\left(\Theta_{X}\right)<3 c_{2}-K^{2}-\Gamma^{2}+$ $2 N$.

REMARK 3.11. One can give a straightforward upper bound on $-\Gamma^{2}$ in terms of geometry of the irreducible components of $\Gamma$ : write the decomposition $\Gamma=\sum_{i} m_{i} C_{i}$ into reduced irreducible components $C_{i}$. The adjunction formula applied to the component $C_{i}$ gives $K . C_{i}+C_{i}^{2} \geq 2 g_{i}-2$, where $g_{i}=g\left(\tilde{C}_{i}\right)$ is the genus of the normalization $\tilde{C}_{i}$ of $C_{i}$. This together with

$$
-\Gamma^{2}=-\sum_{i} m_{i}^{2} C_{i}^{2}-\sum_{i \neq j} m_{i} m_{j} C_{i} \cdot C_{j}
$$

yield

$$
-\Gamma^{2} \leq \sum_{i} m_{i}^{2} K \cdot C_{i}-\sum_{i} m_{i}^{2}\left(2 g_{i}-2\right)-\sum_{i \neq j} m_{i} m_{j} C_{i} \cdot C_{j}
$$

The divisor $2 \Gamma$ is a component of $E_{1}$ in Corollary 1.7. This implies that K. $\Gamma<$ $c\left(3 c_{2}-K^{2}\right)$, where $c=1$, if $r_{1}=3$ and $c=2$, if $r_{1}=2$. From this we obtain $m_{i} K . C_{i}<c\left(3 c_{2}-K^{2}\right)-\sum_{j \neq i} m_{j} K . C_{j}$. Substituting into (3.24) we obtain

$-\Gamma^{2}<c\left(\sum_{i} m_{i}\right)\left(3 c_{2}-K^{2}\right)-\sum_{i} m_{i}^{2}\left(2 g_{i}-2\right)-\sum_{i \neq j} m_{i} m_{j} C_{i} \cdot C_{j}-\sum_{i \neq j} m_{i} m_{j} K . C_{j}$.

This implies

$$
-\Gamma^{2}<c\left(\sum_{i} m_{i}\right)\left(3 c_{2}-K^{2}\right)+2 \sum_{i}^{\circ} m_{i}^{2}
$$

where the second sum is taken over all rational components of $\Gamma$. In the next section we will derive an upper bound on $-\Gamma^{2}$ using the technique of extension construction.

4. More on locally supported moduli. In this section we consider more closely a one-dimensional extension corresponding to an element in $V_{0} \backslash V_{0}^{\prime \prime}$ (see (3.5) for notation).

Fix a cohomology class $\xi$ in $V_{0} \backslash V_{0}^{\prime \prime} \subset H^{1}\left(\Theta_{X}\right)$ and consider the corresponding extension

$$
0 \longrightarrow \mathcal{O}_{X} \longrightarrow \mathcal{T}_{\xi} \longrightarrow \Omega_{X} \longrightarrow 0
$$


This one-dimensional extension is related to the extension $\mathcal{T}_{V_{0}}$ in (2.2) by a diagram analogous to the one in (3.8)

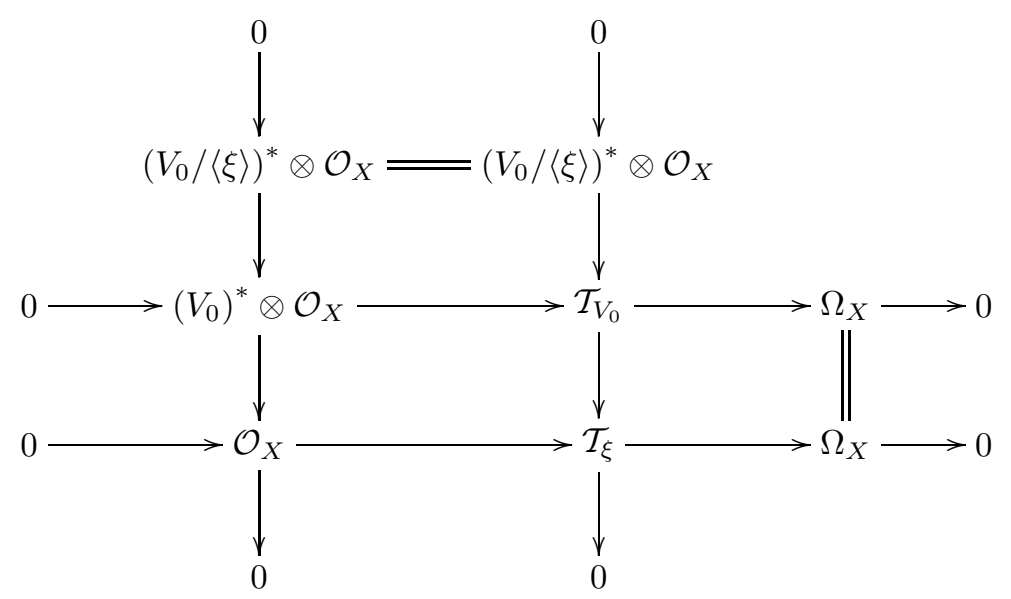

where $\langle\xi\rangle$ is the one-dimensional subspace of $H^{1}\left(\Theta_{X}\right)$ spanned by $\xi$. The morphism $\tilde{\mu}: \mathcal{G} \longrightarrow \mathcal{T}_{V_{0}}$ in $(2.2)$ composed with the morphism $\mathcal{T}_{V_{0}} \longrightarrow \mathcal{T}_{\xi}$ in the above diagram yields the following

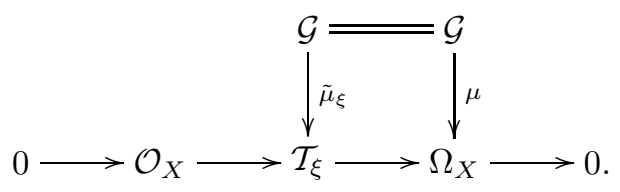

Factoring out by the torsion of the $\operatorname{coker}\left(\tilde{\mu}_{\xi}\right)$ we obtain the following

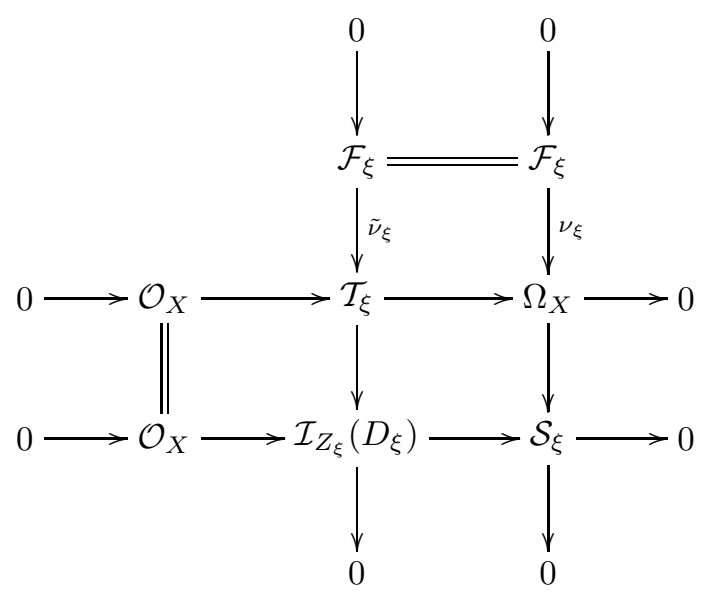

where $\mathcal{F}_{\xi}$ is locally free, $\mathcal{I}_{Z_{\xi}}$ is the sheaf of ideals of some 0 -dimensional subscheme $Z_{\xi}$ on $X$ and $D_{\xi}$ is a nonzero effective divisor.

REMARK 4.1. By an argument analogous to the one in the proof of Lemma 3.5 we obtain that for a general choice of $\xi \in V_{0} \backslash V_{0}^{\prime \prime}$ the divisor $D_{\xi}=\Gamma+E_{2}$, where the components $\Gamma$ and $E_{2}$ are as in (3.10) while $c_{1}\left(\operatorname{Tor}\left(\operatorname{coker}\left(\tilde{\mu}_{\xi}\right)\right)\right)=\Gamma$. In particular, this implies

$$
L=c_{1}(\mathcal{G})=K-D_{\xi}-\Gamma=K-E_{2}-2 \Gamma .
$$


We will now consider the properties of the divisor $D_{\xi}$. For this we take the second exterior power of (4.1)

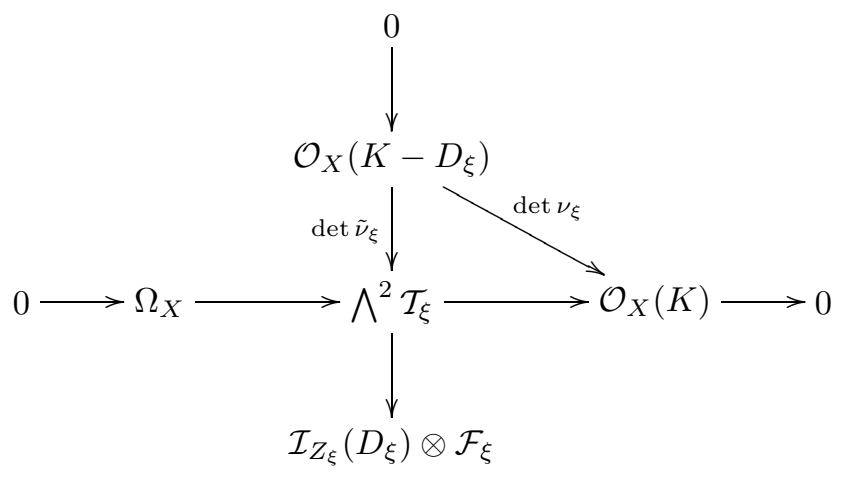

The divisor $D_{\xi}$ is the subscheme where $\operatorname{det} \nu_{\xi}$ vanishes. This implies that the restriction of $\operatorname{det} \tilde{\nu}_{\xi}$ to any reduced irreducible component $C$ of $D_{\xi}$ factors through $\Omega_{X} \otimes \mathcal{O}_{C}$, i.e. we obtain a nonzero morphism $\mathcal{O}_{C}\left(K-D_{\xi}\right) \longrightarrow \Omega_{X} \otimes \mathcal{O}_{C}$. Tensoring with $\mathcal{O}_{X}(-K)$ gives a nonzero morphism $s_{C}: \mathcal{O}_{C}\left(-D_{\xi}\right) \longrightarrow \Theta_{X} \otimes \mathcal{O}_{C}$ and its zero-locus is $Z_{\xi}^{C}=Z_{\xi} \cap C$.

REMARK 4.2. The above considerations can be applied to any component $D$ of $D_{\xi}$. This gives a nonzero morphism

$$
s_{D}: \mathcal{O}_{D}\left(-D_{\xi}\right) \longrightarrow \Theta_{X} \otimes \mathcal{O}_{D}
$$

To understand properties of the morphism $s_{C}$ we combine it with the normal sequence for $C \subset X$ :

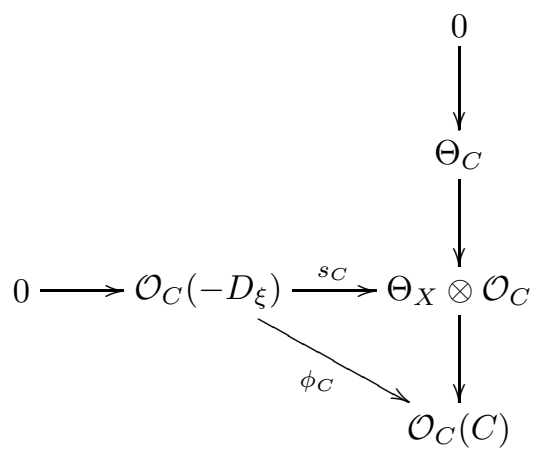

We distinguish two types of irreducible components in $D_{\xi}$ according to whether the morphism $\phi_{C}$ in (4.3) is zero or not.

Type I. $\phi_{C} \neq 0$. Then the zero-locus $Z_{\xi}^{C}$ of $s_{C}$ is contained in the zero-locus of $\phi_{C}$. This implies

$$
\operatorname{deg} Z_{\xi}^{C} \leq C^{2}+C \cdot D_{\xi}
$$

Type II. $\phi_{C}=0$. Then $s_{C}$ factors through $\Theta_{C}$. Let $\eta_{C}: \tilde{C} \longrightarrow C$ be the normalization of $C$. Then we have the following exact sequence of sheaves on $\tilde{C}$ : 


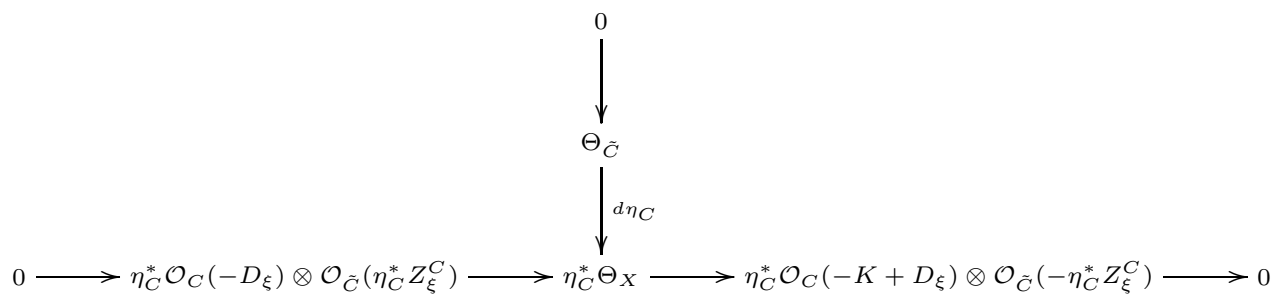

We claim that the injective morphism in the horizontal sequence of (4.5) factors through $\Theta_{\tilde{C}}$. Indeed, the normalization morphism gives $\eta_{C *}: \mathcal{O}_{C} \longrightarrow$ $\eta_{C *} \mathcal{O}_{\tilde{C}}$. Tensoring it with $\Theta_{X}$ and combining with the differential of $\eta_{C}$ gives the following

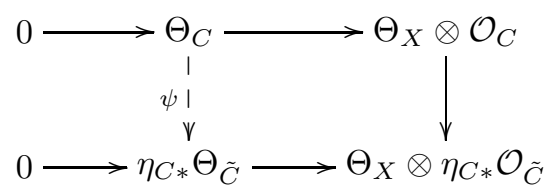

where the induced morphism $\Theta_{C} \longrightarrow \Theta_{X} \otimes \eta_{C *} \mathcal{O}_{\tilde{C}}$ factors through $\eta_{C *} \Theta_{\tilde{C}}$ (see Theorem 11.9,[5]) as indicated in the above diagram. The natural isomorphism $\operatorname{Hom}_{\mathcal{O}_{C}}\left(\Theta_{C}, \eta_{C *} \Theta_{\tilde{C}}\right)=\operatorname{Hom}_{\mathcal{O}_{\tilde{C}}}\left(\eta_{C}^{*} \Theta_{C}, \Theta_{\tilde{C}}\right)$ implies that the morphism $\eta_{C}^{*} \Theta_{C} \longrightarrow \eta_{C}^{*} \Theta_{X}$ factors through $\Theta_{\tilde{C}}$. On the other hand $\eta_{C}^{*}\left(s_{C}\right): \eta_{C}^{*} \mathcal{O}_{C}\left(-D_{\xi}\right) \longrightarrow \eta_{C}^{*} \Theta_{X}$ factors through $\eta_{C}^{*} \Theta_{C}$. So we deduce that $\eta_{C}^{*}\left(s_{C}\right)$ factors through $\Theta_{\tilde{C}}$ as claimed. Thus the diagram (4.5) has the following form

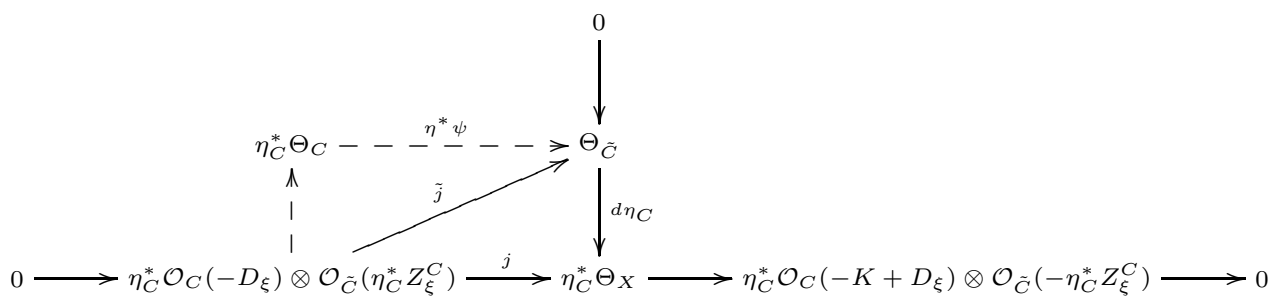

(4.6)

Since cokernel of $j$ in the above diagram is locally free it follows that $\tilde{j}$ is an isomorphism as well as all the oblique arrows in (4.6). This implies

$$
\operatorname{deg} Z_{\xi}^{C} \leq \operatorname{deg}\left(\eta_{C}^{*}\left(Z_{\xi}^{C}\right)\right)=C \cdot D_{\xi}-\left(2 g_{\tilde{C}}-2\right)
$$

where $g_{\tilde{C}}$ is the genus of the normalization of $C$.

REMARK 4.3. The fact that $\tilde{j}$ is an isomorphism and $j$ is injective implies that the differential $d \eta_{C}$ in (4.6) is of maximal rank everywhere, i.e. the morphism $\eta_{C}$ : $\tilde{C} \longrightarrow C \subset X$ is an immersion.

Let us decompose the divisor $D_{\xi}$ as follows

$$
D_{\xi}=D_{\xi}^{\prime}+D_{\xi}^{\prime \prime}
$$


where $D_{\xi}^{\prime}$ (resp. $D_{\xi}^{\prime \prime}$ ) is the part of $D_{\xi}$ composed of the reduced irreducible components of type I (resp. type II). We will be concerned with the part $D_{\xi}^{\prime \prime}$. Let $\left(D_{\xi}^{\prime \prime}\right)_{\text {red }}$ be the reduced part of $D_{\xi}^{\prime \prime}$.

Lemma 4.4. The singular locus of $\left(D_{\xi}^{\prime \prime}\right)_{\text {red }}$ is contained in $Z_{\xi} \cap D_{\xi}^{\prime \prime}$.

Proof. The morphism $\mathcal{O}_{\left(D_{\xi}^{\prime \prime}\right)_{\text {red }}}\left(-D_{\xi}\right) \longrightarrow \Theta_{\left(D_{\xi}^{\prime \prime}\right)_{\text {red }}}$ induced by the morphism $s_{\left(D_{\xi}^{\prime \prime}\right)_{\text {red }}}$ is an isomorphism away from $Z_{\xi} \cap D_{\xi}^{\prime \prime}$. This implies that $\Theta_{\left(D_{\xi}^{\prime \prime}\right)_{\text {red }}, p}$ is a locally free $\mathcal{O}_{\left(D_{\xi}^{\prime \prime}\right)_{\text {red }}, p}$-module for any $p \in\left(D_{\xi}^{\prime \prime}\right)_{\text {red }} \backslash Z_{\xi}$. By a result of Lipman,[11], this implies that $p$ is a smooth point of $\left(D_{\xi}^{\prime \prime}\right)_{\text {red }}$.

Lemma 4.5. Let $C$ be a reduced irreducible curve in $X$ such that $C . D_{\xi}<0$. Then $C$ is a smooth rational curve with C. $D_{\xi}=-2$ or -1 .

Proof. The assumption $C . D_{\xi}<0$ implies that $C$ is a component of $D_{\xi}$ with $C^{2}<$ 0. This implies that $C$ is of type II. From (4.7) and C.D $D_{\xi}<0$ it follows that $g_{\tilde{C}}=0$ and $C . D_{\xi}=-1$ or -2 . This yields that $\operatorname{deg}\left(\eta_{C}^{*}\left(Z^{C}\right)\right) \leq 1$. So $Z^{C}$ must be contained in the smooth locus of $C$ and $s_{C}$ induces an isomorphism $\mathcal{O}_{C}\left(Z^{C}-D_{\xi}\right) \cong \Theta_{C}$. Hence $\Theta_{C}$ is locally free. Again Lipman's result in [11] gives the smoothness of $C$.

The above lemma implies the following.

Proposition 4.6. If $X$ has no smooth rational curves then the divisor $D_{\xi}$ is nef.

REMARK 4.7. If the subspace $V_{0}^{\prime \prime} \neq 0$ and we take the extension $\mathcal{T}_{\xi}$ corresponding to a nonzero $\xi \in V_{0}^{\prime \prime}$, then the divisor $D_{\xi}$ is a component of $\Gamma$ (see (3.2) for notation) and we will denote it by $\Gamma_{\xi}$. The results of Lemma 4.5 and Lemma 3.2 imply that the irreducible curves intersecting $\Gamma_{\xi}$ negatively are smooth rational curves having intersection -1 or -2 with $\Gamma_{\xi}$. In particular, if $X$ has no smooth rational curves then $V_{0}^{\prime \prime}=0$ and $V_{0}=V_{0}^{\prime}$. This combined with Corollary 3.8 yields

$$
\operatorname{dim} V_{0}<\left\{\begin{array}{ll}
2 \alpha\left(3 c_{2}-K^{2}\right)-\Gamma^{2}-\frac{1}{6}\left(1+2 \frac{K \cdot E_{2}^{\prime}}{K^{2}}+\frac{K \cdot F}{K^{2}}\right) K . F, & \text { if } r_{1}=3 \\
3 c_{2}-K^{2}-\Gamma^{2}, & \text { if } r_{1}=2
\end{array} .\right.
$$

We will now apply the above considerations to obtain an upper bound for $\left(-\Gamma^{2}\right)$ in the inequality of Corollary 3.10 (compare with Remark 3.11).

First we recall that by Remark 4.1 the divisor $D_{\xi}=\Gamma+E_{2}$ for a general $\xi \in V_{0} \backslash V_{0}^{\prime \prime}$. Let $N_{2}$ be the number of rational curves (counted with their multiplicities) subject to Lemma 4.5 and contained in $E_{2}$. Then we have the following bound on $-\Gamma^{2}$.

Lemma 4.8. Let $\Gamma, E_{2}$ and $N_{2}$ be as above. Then the following inequality holds.

$$
-\Gamma^{2} \leq\left(\Gamma+E_{2}\right)^{2}-K . \Gamma+2 N_{2} .
$$


Proof. From Lemma 4.5 it follows $D_{\xi} \cdot E_{2}=\left(\Gamma+E_{2}\right) \cdot E_{2} \geq-2 N_{2}$. From (4.2) and Lemma 3.1 we obtain that $\left(2 \Gamma+E_{2}\right) \cdot \Gamma \geq K . \Gamma$. Taking the sum of these two inequalities we obtain

$$
\Gamma^{2}+\left(\Gamma+E_{2}\right)^{2} \geq K \cdot \Gamma-2 N_{2} .
$$

Hence the assertion of the lemma.

Corollary 4.9. Let $X, \Gamma, N$ be as in Corollary 3.10 and let $E_{2}, N_{2}$ be as in Lemma 4.8 then the following holds.

$$
h^{1}\left(\Theta_{X}\right) \leq\left\{\begin{array}{ll}
\frac{3}{2}\left(3 c_{2}-K^{2}\right)+2\left(N+N_{2}\right), & \text { if } r_{1}=3 \\
5\left(3 c_{2}-K^{2}\right)+2\left(N+N_{2}\right)-K . E_{1}-K . \Gamma, & \text { if } r_{1}=2
\end{array} .\right.
$$

Proof. By Hodge index $\left(\Gamma+E_{2}\right)^{2} \leq \frac{\left(\left(\Gamma+E_{2}\right) \cdot K\right)^{2}}{K^{2}}$. Using the fact that $\Gamma+E_{2}$ is a component of $E_{1}$ in Corollary 1.7 and arguing as in the beginning of the proof of Corollary 3.8 we have

$$
\begin{aligned}
\left(\Gamma+E_{2}\right)^{2} \leq \frac{\left(\left(\Gamma+E_{2}\right) \cdot K\right)^{2}}{K^{2}} & \leq \frac{\left(K \cdot E_{1}-K \cdot(F+\Gamma)\right)^{2}}{K^{2}} \\
& \leq \frac{\left(K \cdot E_{1}\right)^{2}}{K^{2}}-2 \frac{K \cdot\left(\Gamma+E_{2}\right)}{K^{2}} K \cdot(F+\Gamma)-\frac{(K \cdot(\Gamma+F))^{2}}{K^{2}} .
\end{aligned}
$$

Substituting this in the inequality of Lemma 4.8 we obtain

$$
-\Gamma^{2} \leq \frac{\left(K \cdot E_{1}\right)^{2}}{K^{2}}-2 \frac{K \cdot\left(\Gamma+E_{2}\right)}{K^{2}} K \cdot(F+\Gamma)-\frac{(K \cdot(\Gamma+F))^{2}}{K^{2}}-K \cdot \Gamma+2 N_{2} .
$$

If $r_{1}=3$ then substituting (4.9) in (3.20) and treating the cases according to the properties of the linear system $|F|$ as it is done in the proof of Corollary 3.10 we obtain

$$
h^{1}\left(\Theta_{X}\right)< \begin{cases}(14 \alpha-4)\left(3 c_{2}-K^{2}\right)+2\left(N+N_{2}\right)+1, & \text { if } \mathrm{F}=0, \\ (14 \alpha-4)\left(3 c_{2}-K^{2}\right)+2\left(N+N_{2}\right), & \text { if }|F| \text { is not composed } \\ & \text { of a pencil, } \\ \left(14 \alpha-4+\frac{1}{6}\right)\left(3 c_{2}-K^{2}\right)+2\left(N+N_{2}\right)+1, & \text { if }|F| \text { is composed of } \\ & \text { a pencil. }\end{cases}
$$

This together with $\alpha \leq \frac{3}{8}$ give $h^{1}\left(\Theta_{X}\right) \leq \frac{5}{4}\left(3 c_{2}-K^{2}\right)+2\left(N+N_{2}\right)$ in the first two cases and $h^{1}\left(\Theta_{X}\right)<\frac{17}{12}\left(3 c_{2}-K^{2}\right)+2\left(N+N_{2}\right)+1$ in the last case. These two inequalities clearly imply

$$
h^{1}\left(\Theta_{X}\right) \leq \frac{3}{2}\left(3 c_{2}-K^{2}\right)+2\left(N+N_{2}\right) .
$$

If $r_{1}=2$ then we use (1.16) to obtain

$$
\left(\Gamma+E_{2}\right)^{2}<4\left(3 c_{2}-K^{2}\right)-K . E_{1} .
$$


This combined with Lemma 4.8 yields

$$
-\Gamma^{2}<4\left(3 c_{2}-K^{2}\right)+2 N_{2}-K . E_{1}-K . \Gamma .
$$

Substituting this in the inequality of Corollary 3.10 we obtain

$$
h^{1}\left(\Theta_{X}\right)<5\left(3 c_{2}-K^{2}\right)+2\left(N+N_{2}\right)-K . E_{1}-K . \Gamma .
$$

$\square$

Corollary 4.10. 1) If the canonical divisor $K$ of $X$ in Corollary 4.9 is ample then

$$
h^{1}\left(\Theta_{X}\right) \leq\left\{\begin{array}{ll}
\frac{21}{4}\left(3 c_{2}-K^{2}\right), & \text { if } r_{1}=3 \\
9\left(3 c_{2}-K^{2}\right)-3 K . \Gamma, & \text { if } r_{1}=2
\end{array} .\right.
$$

In particular, $h^{1}\left(\Theta_{X}\right) \leq 9\left(3 c_{2}-K^{2}\right)$.

2) If $X$ in Corollary 4.9 does not contain smooth rational curves then

$$
h^{1}\left(\Theta_{X}\right) \leq\left\{\begin{array}{ll}
\frac{3}{2}\left(3 c_{2}-K^{2}\right), & \text { if } r_{1}=3 \\
5\left(3 c_{2}-K^{2}\right)-K . E_{1}-K . \Gamma, & \text { if } r_{1}=2
\end{array} .\right.
$$

In particular, $h^{1}\left(\Theta_{X}\right) \leq 5\left(3 c_{2}-K^{2}\right)$.

Proof. The ampleness of $K$ implies

$$
N+N_{2} \leq K .\left(\Gamma+E_{2}\right) \leq K \cdot E_{1}-K .(\Gamma+F) .
$$

If $r_{1}=3$ then by Corollary $1.7 N+N_{2}<2\left(3 c_{2}-K^{2}\right)-K .(\Gamma+F)$. Treating the cases according the properties of the linear system $|F|$ as it is done in the proof of Corollary 3.10 we obtain

$$
h^{1}\left(\Theta_{X}\right)< \begin{cases}14 \alpha\left(3 c_{2}-K^{2}\right)+1-2 K . \Gamma, & \text { if } \mathrm{F}=0, \\ 14 \alpha\left(3 c_{2}-K^{2}\right)-2 K .(\Gamma+F), & \text { if }|F| \text { is not composed of } a \\ & \text { pencil, } \\ 14 \alpha\left(3 c_{2}-K^{2}\right)+1-\frac{23}{12} K . F-2 K . \Gamma, & \text { if }|F| \text { is composed of } a \\ & \text { pencil. }\end{cases}
$$

This combined with $\alpha \leq \frac{3}{8}$ implies $h^{1}\left(\Theta_{X}\right) \leq \frac{21}{4}\left(3 c_{2}-K^{2}\right)$.

If $r_{1}=2$ then combining (4.11) with the inequality of Corollary 4.9 we obtain

$$
h^{1}\left(\Theta_{X}\right)<5\left(3 c_{2}-K^{2}\right)+K . E_{1}-3 K . \Gamma .
$$

Using the bound on $K . E_{1}$ in Corollary 1.7 we deduce

$$
h^{1}\left(\Theta_{X}\right)<9\left(3 c_{2}-K^{2}\right)-3 K . \Gamma .
$$

The second part of the corollary follows from Corollary 4.9 and the fact $N=N_{2}=0$ which is guarantied by Remark 4.7 and the absence of smooth rational curves on $X$. 
We close this section by observing that the upper bound for $h^{1}\left(\Theta_{X}\right)$, as a linear function of $\left(3 c_{2}-K^{2}\right)$, can not be improved since for every rational $\alpha$ in the interval ] $\frac{1}{3}, \frac{1}{2}$ [ we have examples of surfaces with Chern numbers $c_{2}, K^{2}$ whose ratio is equal to $\alpha$ and having $h^{1}\left(\Theta_{X}\right)=c\left(3 c_{2}-K^{2}\right)$, for some universal constant $c$.

ExAmPle 4.11. The following construction is due to Sommese (see [17]).

Let $X$ be a smooth minimal surface of general type with $\alpha=\frac{c_{2}}{K^{2}}=\frac{1}{3}$ and a morphism $\pi: X \longrightarrow C$ onto a smooth curve $C$ of genus $g_{C} \geq 1$ (such surfaces were constructed by Hirzebruch, Inoue, Livné; see [9]).

Take $n$ sheeted unramified cover $\tau: C_{n} \longrightarrow C$ with $C_{n}$ connected (this can be done for any $n \geq 1$, since $g_{C} \geq 1$ ) follow it by a 2 sheeted branched cover $\sigma: C_{m, n} \longrightarrow C_{n}$ having $2 m$ branch points (this can be done for any $m \geq 1$ ). Taking the fibre products we obtain

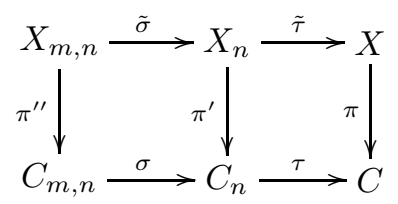

where $X_{n}$ is $n$ sheeted unramified cover of $X$ and $X_{m, n}$ is a double cover of $X_{n}$ branched along $2 m$ fibres of $\pi^{\prime}$ which lie over the branch points of $\sigma$. Choosing the branch points of $\sigma$ away from the critical values of $\pi^{\prime}$ we obtain $X_{m, n}$ smooth and minimal. The Chern invariants of $X_{m, n}$ are as follows:

$$
K_{X_{m, n}}^{2}=2 n K_{X}^{2}+8 m(g-1), c_{2}\left(X_{m, n}\right)=2 n c_{2}(X)+4 m(g-1)
$$

where $g$ is the genus of a smooth fibre of $\pi$. This implies $3 c_{2}\left(X_{m, n}\right)-K_{X_{m, n}}^{2}=$ $4 m(g-1)$ while $h^{1}\left(\Theta_{X_{m, n}}\right)=2 m$, i.e. we obtain surfaces $X$ subject to the following

$$
h^{1}\left(\Theta_{X}\right)=\frac{1}{2(g-1)}\left(3 c_{2}-K^{2}\right) .
$$

Furthermore, as Sommese shows in [17], for any rational $\alpha$ in the interval $] \frac{1}{3}, \frac{1}{2}[$ an appropriate choice of $m$ and $n$ in the above construction gives a surface $X_{m, n}$ with $\alpha_{X_{m, n}}=\alpha$.

5. Surfaces with small values of $3 c_{2}-K^{2}$. In this section we consider surfaces subject to the following conditions:

$$
\begin{array}{cc}
\text { 1) } & \alpha<\frac{1}{2} \\
\text { 2) } & h^{1}\left(\Theta_{X}\right) \geq 2 \\
\text { 3) } & 3 c_{2}-K^{2} \leq \frac{1}{2} \sqrt{K^{2}} .
\end{array}
$$

The last numerical assumption arises naturally in view of the inequality in Corollary 1.7 since combined with the Hodge Index it implies that the intersection pairing is negative semidefinite on the sublattice of $N S(X)$ spanned by the irreducible components of the divisor $E_{1}$ (see Corollary 1.7 for notation). This, as we will see shortly, imposes strong restrictions on the geometry of $E_{1}$ as well as on $X$. 
First we check that the inequality $3 c_{2}-K^{2} \leq \frac{1}{2} \sqrt{K^{2}}$ insures that $\alpha \leq \frac{3}{8}$. Indeed, if $\alpha>\frac{3}{8}$ then $\frac{1}{2} \sqrt{K^{2}} \geq 3 c_{2}-K^{2}>\frac{1}{8} K^{2}$ implying $K^{2}<16$. On the other hand the condition $H^{1}\left(\Theta_{X}\right) \neq 0$ yields $3 c_{2}-K^{2} \geq 4$. This and 3) of (5.1) give $K^{2} \geq 64$. Hence we must have $\alpha \leq \frac{3}{8}$.

From the condition $3 c_{2}-K^{2} \leq \frac{1}{2} \sqrt{K^{2}}$ and $K^{2} \geq 64$ it also follows that the "topological" bound in Proposition 1.1 is $\frac{1}{1-2 \alpha}-2 \leq \frac{10}{7}$. Hence the Bogomolov-Gieseker inequality fails for the extension bundle $\mathcal{T}$ in (1.1) as soon as $h^{1}\left(\Theta_{X}\right) \geq 2$. So the conditions (5.1) imply the decomposition of the canonical divisor $K_{X}=L_{1}+E_{1}$ as in Corollary 1.7 with

$$
E_{1} . K<\left\{\begin{array}{ll}
2\left(3 c_{2}-K^{2}\right) \leq \sqrt{K^{2}}, & r_{1}=3 \\
\frac{8\left(3 c_{2}-K^{2}\right)}{1+\sqrt{1+16(3 \alpha-1)}} \leq \frac{4 \sqrt{K^{2}}}{1+\sqrt{1+\frac{8}{\sqrt{K^{2}}}}}<2 \sqrt{K^{2}}, & r_{1}=2
\end{array} .\right.
$$

Lemma 5.1. Let $E$ be a component of $E_{1}$. Then $E^{2} \leq 0$.

Proof. If $r_{1}=3$ then the first inequality in (5.2) implies that $E . K<\sqrt{K^{2}}$. This combined with the Hodge Index implies $E^{2} \leq 0$.

If $r_{1}=2$ then following through the same argument we obtain $E^{2} \leq 3$. To improve this estimate we return to the inequality (1.15) rewriting it as follows

$$
2\left(3 c_{2}-K^{2}\right)>E_{1} \cdot K+3 \frac{\left(E_{1} \cdot K\right)^{2}}{K^{2}}-2 E_{1}^{2} .
$$

This together with $3 c_{2}-K^{2} \leq \frac{1}{2} \sqrt{K^{2}}$ and $E_{1}^{2} \leq 3$ imply $E_{1} . K<\sqrt{K^{2}}+3$. Using the Hodge Index and $K^{2} \geq 64$ we obtain $E_{1}^{2} \leq 1$. Substituting this in (5.3) yields $E_{1} . K<\sqrt{K^{2}}$. This implies $E . K<\sqrt{K^{2}}$ and $E^{2} \leq 0$.

In what follows we consider the geometric consequences of Lemma 5.1.

Corollary 5.2. $E_{1} . K \leq 3 c_{2}-K^{2}$.

Proof. The definition of $K_{X}$-destabilizing filtration in (1.2) and the notation in (1.3) imply $E_{1}=\sum_{i \geq 2} L_{i}$. From the proof of Lemma 5.1 and Remark 1.4 it follows that $L_{i} . K<\sqrt{K^{2}}$, for every $i \geq 2$. This combined with the Hodge index yields $L_{i}^{2} \leq 0$, for every $i \geq 2$. Substituting in (1.7) we obtain

$$
2 c_{2} \geq 2 d_{1}-L_{1}^{2}+K^{2}
$$

We know, by Corollary 1.7, that the rank of $\mathcal{T}_{1}$ is 2 or 3 . In both cases we obtain $d_{1} \geq \frac{1}{3} L_{1}^{2}$ (see the proof of Corollary 1.7 for details). Substituting in (5.4) yields

$$
2 c_{2} \geq-\frac{1}{3} L_{1}^{2}+K^{2}=-\frac{1}{3}\left(K-E_{1}\right)^{2}+K^{2}=\frac{2}{3} K^{2}+\frac{2}{3} K \cdot E_{1}-\frac{1}{3} E_{1}^{2} .
$$

This and Lemma 5.1 imply $E_{1} . K \leq 3 c_{2}-K^{2}$. $\square$ 
Proposition 5.3. Let $r_{1}=3$ and $c_{1}(\mathcal{K}) \neq 0$, where the sheaf $\mathcal{K}$ is as in (1.11). Then the line bundle $\mathcal{K}=\mathcal{O}_{X}(F)$ is generated by global sections and defines a morphism

$$
f: X \longrightarrow B
$$

where $B$ is a smooth projective curve and $f$ is a surjective morphism with connected fibres. Let $F_{0}$ be the class of a smooth fibre of $f$ and write $F=f^{*}(Z)$ for some divisor $Z$ on $B$. Then $F=(\operatorname{deg} Z) F_{0}$ and $(\operatorname{deg} Z) F_{0} . K \leq 3 c_{2}-K^{2}$.

Proof. We already know that $\mathcal{O}_{X}(F)$ has at most 0-dimensional base locus (see a) of the proof of Corollary 1.7). From (1.13) it also follows that $F$ is a component of $E_{1}$. This and Lemma 5.1 imply that $F^{2} \leq 0$. Since $F$ is nef we conclude that $F^{2}=0$. This also implies that $\mathcal{O}_{X}(F)$ is generated by global sections and the image of the morphism defined by $\mathcal{O}_{X}(F)$ is a curve. Taking its normalization and then Stein factorization we obtain the asserted morphism.

The last inequality of the proposition follows from the bound on $E_{1} . K$ in Corollary 5.2 .

Next we turn to the case when $r_{1}=3$ and $\mathcal{K}=\mathcal{O}_{X}$ or $r_{1}=2$. In this case the subspace $V_{0}$ of locally supported moduli of $X$ is of codimension at least 1 in $H^{1}\left(\Theta_{X}\right)$. Thus $V_{0}$ is nonzero and we consider the extension $\mathcal{T}_{\xi}$ for a general $\xi$ in $V_{0}$. This gives rise to the divisor $D_{\xi}$ which is a nonempty component of $E_{1}$ with $D_{\xi}^{2} \leq 0$ and $D_{\xi} \cdot K \leq 3 c_{2}-K^{2}$ (Lemma 5.1 and Corollary 5.2, respectively). We decompose $D_{\xi}$ into two parts

$$
D_{\xi}=D_{\xi}^{\prime}+D_{\xi}^{\prime \prime}
$$

according to type as in (4.8).

Lemma 5.4. Assume $X$ has no smooth rational curves. Then $D_{\xi}^{2}=\left(D_{\xi}^{\prime}\right)^{2}=$ $\left(D_{\xi}^{\prime \prime}\right)^{2}=0$ and the divisors $D_{\xi}^{\prime}$ and $D_{\xi}^{\prime \prime}$ have the following properties.

$\left(i^{\prime}\right)$ Each irreducible component $C$ of $D_{\xi}^{\prime}$ is smooth with $\Omega_{X} \otimes \mathcal{O}_{C}=\mathcal{O}_{C}(-C) \oplus$ $\mathcal{O}_{C}(K+C)$,

$\left(i i^{\prime}\right)$ for any two curves $C$ and $C^{\prime}$ in $D_{\xi}^{\prime}$ the intersection $C . C^{\prime}=0$,

$\left(\right.$ iii') Let $m_{C}$ be the multiplicity of the component $C$ in $D_{\xi}^{\prime}$. Then $\mathcal{O}_{C}\left(\left(m_{C}+1\right) C\right)=$ $\mathcal{O}_{C}$, i.e. $\mathcal{O}_{C}(C)$ is a torsion point of $\operatorname{Pic}^{\circ}(C)$ and its order of torsion $t_{C}$ divides $\left(m_{C}+1\right)$.

$\left(i^{\prime \prime}\right)$ Each irreducible component $C$ of $D_{\xi}^{\prime \prime}$ is a rational curve with a single double point,

$\left(i i^{\prime \prime}\right)$ any two distinct irreducible components of $D_{\xi}^{\prime \prime}$ are either disjoint or intersect at their (common) double point.

$\left(\right.$ iii" $\left.{ }^{\prime \prime}\right)$ Let $p_{1}, \ldots, p_{M^{\prime \prime}}$ be the set of distinct doubles points as above. Then the decomposition $D_{\xi}^{\prime \prime}=\sum_{i}^{M^{\prime \prime}} D_{\xi}^{\prime \prime}\left(p_{i}\right)$ of $D_{\xi}^{\prime \prime}$ into connected components is such that every connected component $D_{\xi}^{\prime \prime}\left(p_{i}\right)=\sum_{j=1}^{M_{i}^{\prime \prime}} m_{i j} C_{i j}$, where $C_{i j}$ 's are rational curves with a single double point at $p_{i}$. 
Proof. Since $X$ has no smooth rational curves Proposition 4.6 implies that $D_{\xi}$ is nef. This yields $D_{\xi} . C \geq 0$ for any reduced irreducible component $C$ of $D_{\xi}$. From this it follows $D_{\xi}^{2} \geq 0$ which combined with Lemma 5.1 gives $D_{\xi}^{2}=0$ and $D_{\xi} \cdot C=0$ for any reduced irreducible component $C$ of $D_{\xi}$.

Let $C$ be a reduced irreducible component of $D_{\xi}^{\prime}$. Then the inequality (4.4) combined with $C^{2} \leq 0$ and $C . D_{\xi}=0$ yields $C^{2}=0$ and the morphism $\phi_{C}$ in (4.3) must be an isomorphism. This implies the assertions $\left(i^{\prime}\right)-\left(i i i^{\prime}\right)$ as well as $\left(D_{\xi}^{\prime}\right)^{2}=$ $D_{\xi}^{\prime} \cdot D_{\xi}^{\prime \prime}=\left(D_{\xi}^{\prime \prime}\right)^{2}=0$.

We turn now to the components of $D_{\xi}^{\prime \prime}$. From the above argument we deduce that $C . D_{\xi}^{\prime \prime}=0$ for every reduced irreducible component $C$ of $D_{\xi}^{\prime \prime}$. This together with (4.7) implies $g_{\tilde{C}} \leq 1$. We claim that $g_{\tilde{C}}=0$. In fact, if $g_{\tilde{C}}=1$ then (4.7) implies that the the zero-locus of the morphism $s_{C}$ in (4.3) is empty. This and Lemma 4.4 yield that $C$ is a smooth curve of genus 1 and $C$ does not meet any other component of $D_{\xi}^{\prime \prime}$. This combined with $\left(D_{\xi}^{\prime \prime}\right) . C=0$ yields $C^{2}=0$. By the adjuction formula for $C$ we obtain $K . C=C^{2}=0$ which contradicts the fact that $X$ is a minimal surface of general type. Thus $C$ is a rational curve. By assumption it can not be smooth. From (4.7) and Remark 4.3 it follows that $Z_{\xi}^{C}$ must be a double point of $C$. This proves $\left(i^{\prime \prime}\right)$ of the lemma.

From Lemma 4.4 it follows that if two distinct irreducible components of $D_{\xi}^{\prime \prime}$ intersect then they intersect at their (common) double point. Hence the assertion $\left(i i^{\prime \prime}\right)$ of the lemma.

Turning to $\left(i i i^{\prime \prime}\right)$ we put $p_{1}, \ldots, p_{M^{\prime \prime}}$ to be distinct double points as above. Then $\left(i i i^{\prime \prime}\right)$ follows from $\left(i^{\prime \prime}\right)$ and $\left(i i^{\prime \prime}\right)$.

Proposition 5.5. Let $D_{\xi}^{\prime}$ and $D_{\xi}^{\prime \prime}$ be as in Lemma 5.4 and let $M^{\prime}$ (resp. $M^{\prime \prime}$ ) be the number of connected components of $D_{\xi}^{\prime}\left(\right.$ resp. $\left.D_{\xi}^{\prime \prime}\right)$. If $M^{\prime}+M^{\prime \prime} \geq 2$, then $X$ admits a morphism

$$
f: X \longrightarrow B
$$

where $B$ is a smooth projective curve and $f$ is a surjective morphism with connected fibres. Furthermore, the divisors $D_{\xi}^{\prime}$ and $D_{\xi}^{\prime \prime}$ are contained in the fibres of $f$. More precisely, let $F_{0}$ be the class of a fibre of $f$, then $F_{0}=t_{C} C$, where $C$ is an irreducible curve in $D_{\xi}^{\prime}$ and $t_{C}$ is as in (iii') of Lemma 5.4, and $F_{0}=\delta_{i} D_{\xi}^{\prime \prime}\left(p_{i}\right)$, where $\delta_{i} \in \mathbf{Q}^{+}$ and $D_{\xi}^{\prime \prime}\left(p_{i}\right)$ is a connected component of $D_{\xi}^{\prime \prime}$ as in $\left(i i^{\prime \prime}\right)$ of Lemma 5.4.

Proof. Consider the set $\left\{C \mid\right.$ irreducible curve in $\left.D_{\xi}^{\prime}\right\} \cup\left\{D_{\xi}^{\prime \prime}\left(p_{i}\right) \mid i=1, \ldots, M^{\prime \prime}\right\}$ (see Lemma 5.4 for notation). From the assumption $M^{\prime}+M^{\prime \prime} \geq 2$ it follows that the set contains at least two distinct and hence disjoint divisors, say $\Sigma_{1}$ and $\Sigma_{2}$. From $\left(\left(K . \Sigma_{1}\right) \Sigma_{2}-\left(K . \Sigma_{2}\right) \Sigma_{1}\right) \cdot K=0$ and the Hodge Index it follows that $\left(\left(K . \Sigma_{1}\right) \Sigma_{2}-\left(K . \Sigma_{2}\right) \Sigma_{1}\right)^{2} \leq 0$. Since $\Sigma_{1} \cdot \Sigma_{2}=\left(\Sigma_{1}\right)^{2}=\left(\Sigma_{2}\right)^{2}=0$ it follows that $\left(K . \Sigma_{1}\right) \Sigma_{2}-\left(K . \Sigma_{2}\right) \Sigma_{1}=0$ in $N S(X)$. This implies that some power of $\mathcal{O}_{X}\left(\left(K . \Sigma_{1}\right) \Sigma_{2}-\left(K . \Sigma_{2}\right) \Sigma_{1}\right)$ lies in the kernel of $H^{1}\left(\mathcal{O}_{X}^{*}\right) \longrightarrow H^{2}(X, \mathbf{Z})$. If $H^{1}\left(\mathcal{O}_{X}\right)=0$, then $\mathcal{L}=\mathcal{O}_{X}\left(m\left(K . \Sigma_{1}\right) \Sigma_{2}\right)=\mathcal{O}_{X}\left(m\left(K . \Sigma_{2}\right) \Sigma_{1}\right)$ for some positive integer $m$. This implies that the divisors $m\left(K . \Sigma_{1}\right) \Sigma_{2}, m\left(K . \Sigma_{2}\right) \Sigma_{1}$ give a base point free pencil in the linear system $|\mathcal{L}|$, i.e. we have a morphism $X \longrightarrow \mathbf{P}^{1}$. Taking the Stein factorization yields the assertion.

We turn now to the case $H^{1}\left(\mathcal{O}_{X}\right) \neq 0$. If $M^{\prime \prime} \neq 0$, then the Albanese map of $X$ contracts the divisors $D_{\xi}^{\prime \prime}\left(p_{i}\right)$ 's to points. From this and $\left(D_{\xi}^{\prime \prime}\left(p_{i}\right)\right)^{2}=0$ it follows that 
the image of the Albanese map is a curve. This gives the asserted morphism. So we may assume $M^{\prime \prime}=0$. Then $M^{\prime} \geq 2$ and we take two distinct curves $C_{1}$ and $C_{2}$ in $D_{\xi}^{\prime}$. Arguing as in the first part of the the proof we obtain that there exist positive integers $a_{1}, a_{2}$ such that $\mathcal{L}=\mathcal{O}_{X}\left(a_{1} C_{1}-a_{2} C_{2}\right) \in P i c^{\circ}(X)$. We may assume that $\mathcal{L}$ is not of finite order (otherwise we are done by the first part of the proof). The restriction $\mathcal{L} \otimes \mathcal{O}_{C_{1}}=\mathcal{O}_{C_{1}}\left(a_{1} C_{1}\right)$ is, by Lemma 5.4, $\left(i i i^{\prime}\right)$, a torsion point of $P i c^{\circ}\left(C_{1}\right)$. Thus the restriction morphism $r_{C_{1}}: P i c^{\circ}(X) \longrightarrow P i c^{\circ}\left(C_{1}\right)$ contains an infinite subgroup of the cyclic group $\left\{\mathcal{L}^{n}\right\}_{n \in \mathbf{Z}}$. Hence $\operatorname{ker}\left(r_{C_{1}}\right)$ is an abelian subvariety of $P i c^{\circ}(X)$ of dimension $\geq 1$. This implies that the kernel of the differential of $r_{C_{1}}$ at 0 is nonzero. But this differential is $H^{1}\left(\mathcal{O}_{X}\right) \longrightarrow H^{1}\left(\mathcal{O}_{C_{1}}\right)$. By Ramanujam's Lemma (see TheoremA, [3]) $n C_{1}$, for some $n \in \mathbf{N}$, moves in an irrational pencil. More precisely, the inclusion $C_{1} \subset X$ gives the morphism of the Albanese varieties $\phi: A l b\left(C_{1}\right) \longrightarrow A l b(X)$ and the image of $\phi$ is a proper abelian subvariety of $\operatorname{Alb}(X)$ since the differential of $\phi$ at 0 is dual to $H^{1}\left(\mathcal{O}_{X}\right) \longrightarrow H^{1}\left(\mathcal{O}_{C_{1}}\right)$. Consider $\psi: X \longrightarrow A l b(X) / \operatorname{Im}(\phi)$. This morphism contracts $C_{1}$ to a point. Since $C_{1}^{2}=0$ the image of $\psi$ must be a curve. This yields the asserted morphism.

From the construction of the morphism $f$ it follows that the class of its fibre $F_{0}$ is a positive rational multiple of either $D_{\xi}^{\prime \prime}\left(p_{i}\right)$ or any irreducible $C$ in $D_{\xi}^{\prime}$. Furthermore, in the latter case Lemma 5.4, $\left(i i^{\prime}\right)$ and Lemma 8.3,III,[1], imply that $C$ is the support of the multiple fibre of multiplicity $t_{C}$, i.e. $t_{C} C=F_{0}$.

Corollary 5.6. Let $X$ be a smooth surface subject to (5.1) and let $V_{0}$ be the subspace of locally supported moduli of $X$. If $X$ contains no rational curves, then

$$
\operatorname{dim} V_{0} \leq \frac{1}{2} K \cdot D_{\xi}
$$

where $D_{\xi}$ is as in Lemma 5.4 and $\xi \in V_{0}$ is general.

Proof. For a general $\xi \in V_{0}$ the subspace $V_{0} \subset H^{1}\left(\Theta_{X}\right)$ is supported on $D_{\xi}$, i.e. $V_{0} \subset \operatorname{ker}\left(H^{1}\left(\Theta_{X}\right) \longrightarrow H^{1}\left(\Theta_{X}\left(D_{\xi}\right)\right)\right)$. This implies that $\operatorname{dim} V_{0} \leq h^{0}\left(\Theta_{X} \otimes\right.$ $\left.\mathcal{O}_{D_{\xi}}\left(D_{\xi}\right)\right)$. Since $X$ has no rational curves Lemma 5.4 implies that $D_{\xi}$ is composed of the irreducible components of type I only, i.e. $D_{\xi}=D_{\xi}^{\prime}$ (see (5.5)). From this it follows

$$
\operatorname{dim} V_{0} \leq h^{0}\left(\Theta_{X} \otimes \mathcal{O}_{D_{\xi}}\left(D_{\xi}\right)\right) \leq \sum_{C} h^{0}\left(\Theta_{X} \otimes \mathcal{O}_{m_{C} C}\left(m_{C} C\right)\right)
$$

where the sum is taken over the reduced irreducible components of $D_{\xi}^{\prime}$.

Let $t_{C}$ be the order of torsion of $\mathcal{O}_{C}(C)$ (see Lemma 5.4, $\left(i i i^{\prime}\right)$ ). Using Lemma $5.4,\left(i^{\prime}\right)$ we obtain

$$
h^{0}\left(\Theta_{X} \otimes \mathcal{O}_{m_{C} C}\left(m_{C} C\right)\right) \leq \begin{cases}\frac{m_{C}+1}{t_{C}}, & \text { if } t_{C} \geq 2 \\ m_{C}, & \text { if } t_{C}=1\end{cases}
$$

This combined with (5.6) yields

$$
\operatorname{dim} V_{0} \leq \sum_{C}^{1} m_{C}+\sum_{C}^{2} \frac{m_{C}+1}{t_{C}}
$$

where the first (resp. second) sum is taken over the reduced irreducible components $C$ of $D_{\xi}$ with $t_{C}=1$ (resp. $t_{C} \geq 2$ ). Observing that $K . C \geq 2$ for every irreducible 
component $C$ of $D_{\xi}$ we obtain

$$
\sum_{C}^{1} m_{C} \leq \frac{1}{2} K \cdot D_{\xi}^{(1)} \text { and } \sum_{C}^{2} \frac{m_{C}}{t_{C}} \leq \frac{1}{4} K \cdot D_{\xi}^{(2)}
$$

where $D_{\xi}^{(1)}$ (resp. $\left.D_{\xi}^{(2)}\right)$ is the part of $D_{\xi}$ composed of the irreducible curves $C$ with $t_{C}=1$ (resp. $t_{C} \geq 2$ ). Furthermore, $t_{C} K . C \geq 4$, for every irreducible curve $C$ in $D_{\xi}^{(2)}$. This yields $\sum_{C}^{2} \frac{1}{t_{C}} \leq \frac{1}{4} K . D_{\xi}^{(2)}$. Combining this with the second inequality in (5.8) gives

$$
\sum_{C}^{2} \frac{m_{C}+1}{t_{C}} \leq \frac{1}{2} K \cdot D_{\xi}^{(2)}
$$

Substituting this and the first inequality in (5.8) into (5.7) we obtain

$$
\operatorname{dim} V_{0} \leq \frac{1}{2} K \cdot D_{\xi}^{(1)}+\frac{1}{2} K \cdot D_{\xi}^{(2)}=\frac{1}{2} K \cdot D_{\xi} .
$$

$\square$

Corollary 5.7. Let $X$ be as in Corollary 5.6. Then

$$
h^{1}\left(\Theta_{X}\right) \leq\left\{\begin{array}{ll}
\frac{1}{2}\left(3 c_{2}-K^{2}\right), & \text { if } r_{1}=2 \\
\frac{1}{2}\left(3 c_{2}-K^{2}\right)+1, & \text { if } r_{1}=3
\end{array} .\right.
$$

Proof. If $r_{1}=2$ then $h^{1}\left(\Theta_{X}\right)=\operatorname{dim} V_{0}$. This combined with Corollary 5.6 and Corollary 5.2 gives $h^{1}\left(\Theta_{X}\right) \leq \frac{1}{2}\left(3 c_{2}-K^{2}\right)$.

If $r_{1}=3$ then $h^{1}\left(\Theta_{X}\right)=\operatorname{dim} V_{0}+\operatorname{dim} V_{1}$ and we consider two case according to properties of the linear system $|F|$, where $F$ is as in Proposition 5.3.

Case: $F=0$. Then $\operatorname{dim} V_{1}=1$ and $h^{1}\left(\Theta_{X}\right)=\operatorname{dim} V_{0}+1$. Arguing as in the case $r_{1}=2$ we obtain $h^{1}\left(\Theta_{X}\right) \leq \frac{1}{2}\left(3 c_{2}-K^{2}\right)+1$.

Case: $F \neq 0$. Then by Proposition 5.3 the linear system $|F|$ defines a fibration $f: X \longrightarrow B$ with the class of a smooth fibre $F_{0}$ and $F=\operatorname{deg}(Z) F_{0}$ for some divisor $Z$ on $B$. In particular, $\operatorname{dim} V_{1} \leq \operatorname{deg}(Z)+1=\frac{K \cdot F}{K \cdot F_{0}}+1$. From this it follows

$$
h^{1}\left(\Theta_{X}\right) \leq \frac{K \cdot F}{K \cdot F_{0}}+1+\operatorname{dim} V_{0}
$$

To bound $\operatorname{dim} V_{0}$ we use the argument in the proof of Corollary 5.6 with the following modification. By Proposition 5.5 the irreducible components $C$ of $D_{\xi}$ are related to the class of the fibre $F_{0}$ as follows: $t_{C} C=F_{0}$, where $t_{C}$ is the order of torsion of $\mathcal{O}_{C}(C)$. Using this relation and the notation in (5.8) we obtain

$$
\sum_{C}^{1} m_{C}+\sum_{C}^{2} \frac{m_{C}}{t_{C}}=\frac{K \cdot D_{\xi}^{(1)}}{K \cdot F_{0}}+\frac{K \cdot D_{\xi}^{(2)}}{K \cdot F_{0}}=\frac{K \cdot D_{\xi}}{K \cdot F_{0}} \text { and } \sum_{C}^{2} \frac{1}{t_{C}}=\sum_{C}^{2} \frac{K \cdot C}{K \cdot F_{0}} \leq \frac{K \cdot D_{\xi}^{(2)}}{K \cdot F_{0}} .
$$


Substituting this in (5.7) we obtain

$$
\operatorname{dim} V_{0} \leq \frac{1}{K \cdot F_{0}}\left(K \cdot D_{\xi}+K \cdot D_{\xi}^{(2)}\right) .
$$

This together with (5.9) yield

$$
\begin{aligned}
h^{1}\left(\Theta_{X}\right) & \leq \frac{1}{K \cdot F_{0}}\left(K \cdot F+K \cdot D_{\xi}+K \cdot D_{\xi}^{(2)}\right)+1 \\
& =\frac{1}{K \cdot F_{0}}\left(K \cdot F+2 K \cdot D_{\xi}-K \cdot D_{\xi}^{(1)}\right)+1 \\
& \leq \frac{1}{K \cdot F_{0}}\left(2 K \cdot E_{1}-K \cdot F-K \cdot D_{\xi}^{(1)}\right)+1
\end{aligned}
$$

where the last inequality follows from the fact that $F+D_{\xi}$ is a component of $E_{1}$ (see (1.13), (2.3), (3.3) and Remark 4.1). Combining (5.11) with Corollary 5.2 we obtain

$$
h^{1}\left(\Theta_{X}\right) \leq \frac{2}{K \cdot F_{0}}\left(3 c_{2}-K^{2}\right)+1-\operatorname{deg}(Z)
$$

where $Z$ is as in Proposition 5.3. Recalling that $K . F_{0} \geq 4$ (see Claim in the proof of Corollary 2.4) we deduce $h^{1}\left(\Theta_{X}\right) \leq \frac{1}{2}\left(3 c_{2}-K^{2}\right) \square$

Proposition 5.8. Let $X$ be a smooth surface subject to (5.1) and let $\Omega_{X}$ be ample. Then $X$ has divisorial moduli only. More precisely, let $\mathcal{O}_{X}(F)$ be as in Proposition 5.3 then there is an isomorphism

$$
H^{0}\left(\mathcal{O}_{X}(F)\right) \stackrel{\sim}{\rightarrow} H^{1}\left(\Theta_{X}\right) .
$$

Furthermore, the $K_{X}$-maximal destabilizing subsheaf $\mathcal{T}_{1}$ is given by the extension

$$
0 \longrightarrow \mathcal{O}_{X}(-F) \longrightarrow \mathcal{T}_{1} \longrightarrow \Omega_{X} \longrightarrow 0
$$

and the cup-product with this extension (viewed as an element of $\left.H^{1}\left(\Theta_{X}(-F)\right)\right)$ defines the isomorphism (5.12).

Proof. The ampleness of $\Omega_{X}$ implies that $X$ has neither rational curves nor the curves subject to $\left(i^{\prime}\right)$ of Lemma 5.4. This yields $V_{0}=0$, i.e. $X$ has the divisorial moduli only and the sheaf $\mathcal{T}_{1}$ in (1.11) fits into the following exact sequence

$$
0 \longrightarrow \mathcal{O}_{X}(-F) \longrightarrow \mathcal{T}_{1} \longrightarrow \mathcal{T}_{1}^{\prime} \longrightarrow 0 \text {. }
$$

Since the linear system $|F|$ is base point free and $F^{2}=0$ we obtain that the sequence (1.12) has the form

$$
0 \longrightarrow \mathcal{R}^{*} \longrightarrow H^{1}\left(\Theta_{X}\right) \otimes \mathcal{O}_{X} \longrightarrow \mathcal{O}_{X}(F) \longrightarrow 0 .
$$

Furthermore, the vanishing of $V_{0}$ implies that the homomorphism

$$
H^{1}\left(\Theta_{X}\right) \longrightarrow H^{0}\left(\mathcal{O}_{X}(F)\right)
$$

arising from the cohomology sequence of (5.15) is injective. Thus we have the first assertion of the proposition. 
In view of the sequence (5.14) the remaining part of the proposition follows as soon as we show that $\mathcal{T}_{1}^{\prime}=\Omega_{X}$ or, equivalently, $c_{1}\left(\mathcal{S}_{1}\right)=0$, where $\mathcal{S}_{1}$ is as in (1.11). In order to do this we consider the extension

$$
0 \longrightarrow V^{*} \otimes \mathcal{O}_{X} \longrightarrow \mathcal{T}_{V} \longrightarrow \Omega_{X} \longrightarrow 0
$$

defined by a general 2-dimensional subspace $V$ of $H^{1}\left(\Theta_{X}\right)$.

The inclusion $\mathcal{T}_{1} \longrightarrow \mathcal{T}$ induces a monomorphism $\mathcal{T}_{1} \longrightarrow \mathcal{T}_{V}$ which gives rise to the following diagram

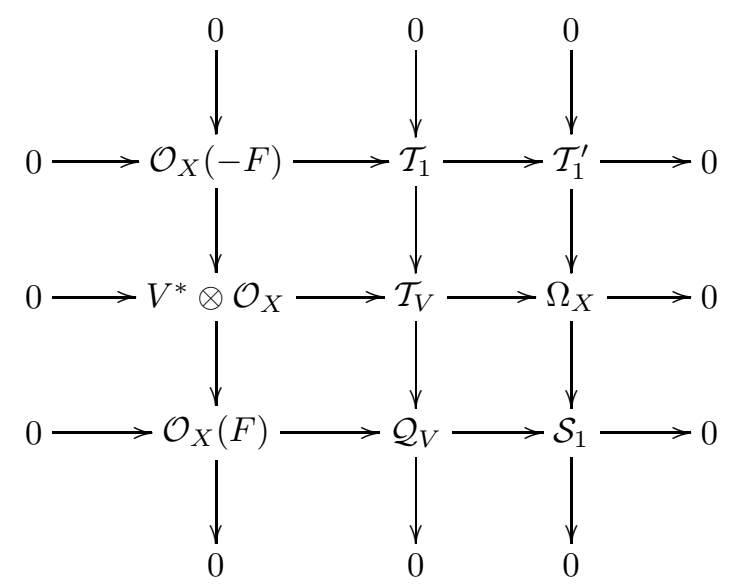

Factoring out by the torsion of $\mathcal{Q}_{V}$ we obtain

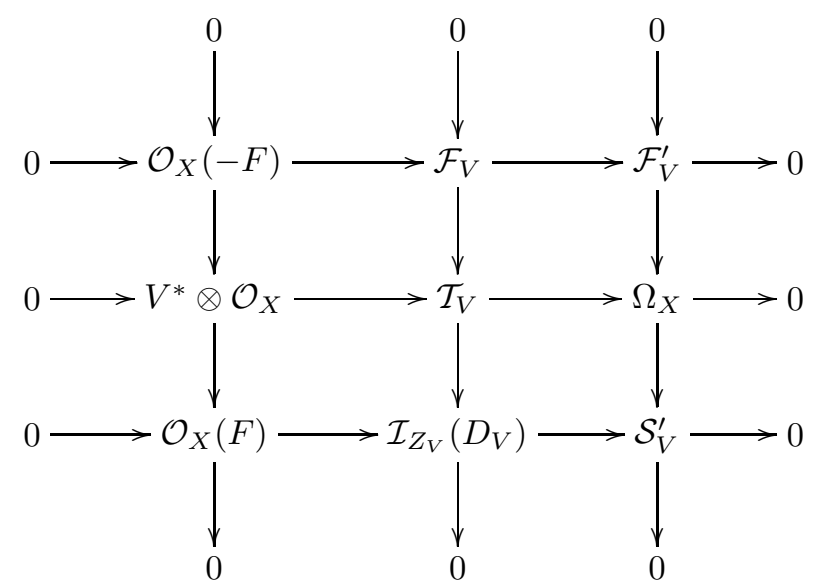

where $\mathcal{I}_{Z_{V}}$ is the sheaf of ideals of some 0 -dimensional subscheme $Z_{V}$ of $X$.

Claim. $\mathcal{F}_{V}^{\prime}=\Omega_{X}$.

Let us assume this and complete the proof of the proposition.

From the claim it follows that the sheaf $\mathcal{F}_{V}$ fits into the following exact sequence

$$
0 \longrightarrow \mathcal{O}_{X}(-F) \longrightarrow \mathcal{F}_{V} \longrightarrow \Omega_{X} \longrightarrow 0 \text {. }
$$

This sequence can be viewed as an (nonzero) element of $\operatorname{Ext}^{1}\left(\Omega_{X}, \mathcal{O}_{X}(-F)\right)=$ $H^{1}\left(\Theta_{X}(-F)\right)$. Denote the corresponding cohomology class in $H^{1}\left(\Theta_{X}(-F)\right)$ by $\tau$. 
The cup-product with $\tau$ gives rise to the linear map

$$
H^{0}\left(\mathcal{O}_{X}(F)\right) \stackrel{\tau}{\longrightarrow} H^{1}\left(\Theta_{X}\right) .
$$

The ampleness of $\Omega_{X}$ guaranties that this homomorphism is injective. Combining this with the injectivity in (5.16) we deduce that the cup-product in (5.21) is an isomorphism. Furthermore, this also implies that $\mathcal{F}_{V}$ lifts to $\mathcal{T}$, i.e. we have a morphism of extensions

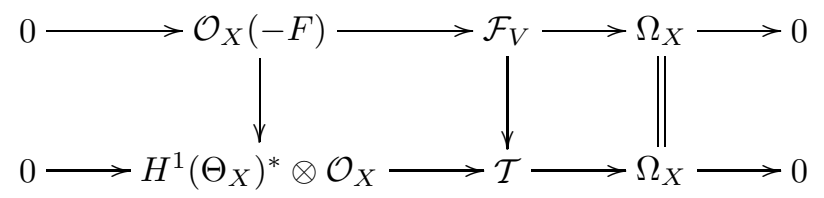

The maximality of $\mathcal{T}_{1}$ yields the equality $\mathcal{T}_{1}=\mathcal{F}_{V}$.

Proof of Claim. Take a nonzero element $\xi$ in $V$ and consider the corresponding one-dimensional extension $\mathcal{T}_{\xi}$

$$
0 \longrightarrow \mathcal{O}_{X} \longrightarrow \mathcal{T}_{\xi} \longrightarrow \Omega_{X} \longrightarrow 0 .
$$

This extension is related to $\mathcal{T}_{V}$ by the following exact sequence

$$
0 \longrightarrow \mathcal{O}_{X} \longrightarrow \mathcal{T}_{V} \longrightarrow \mathcal{T}_{\xi} \longrightarrow 0
$$

Putting this together with vertical sequence in the middle of (5.19) we obtain

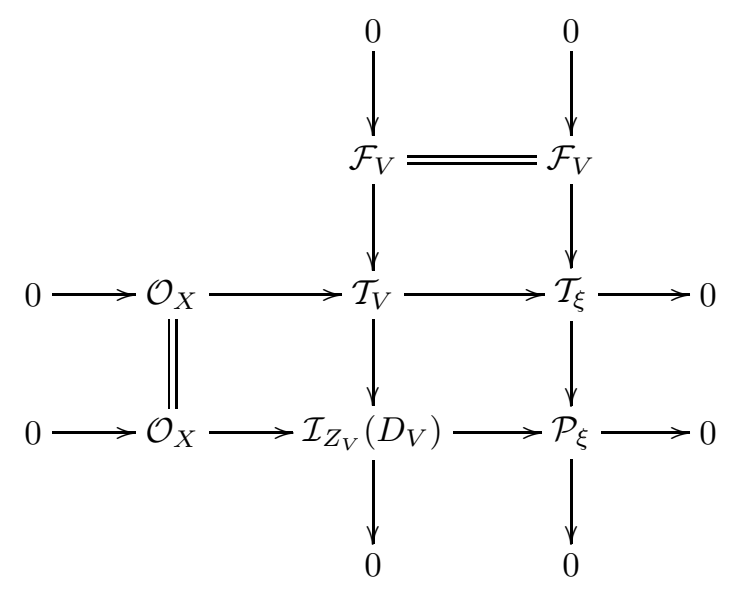

In particular, we obtain a distinguished divisor in the linear system $\left|D_{V}\right|$ which we continue to denote $D_{V}$. From (5.19) we deduce

$$
D_{V}=F_{V}+E_{V}
$$

where $F_{V}$ is the divisor in the linear system $|F|$ corresponding to the line $(V /\langle\xi\rangle)^{*}$ in $V^{*} \subset H^{0}\left(\mathcal{O}_{X}(F)\right)$, and $E_{V}=c_{1}\left(\mathcal{S}_{V}^{\prime}\right)$, where $\mathcal{S}_{V}^{\prime}$ is as in (5.19). We will now establish the following.

(i) $D_{V}$ is nef 
(ii) $Z_{V}=\emptyset$

The argument is essentially the same as in the considerations of (4.1). Namely, we take the third exterior power of $(5.22)$

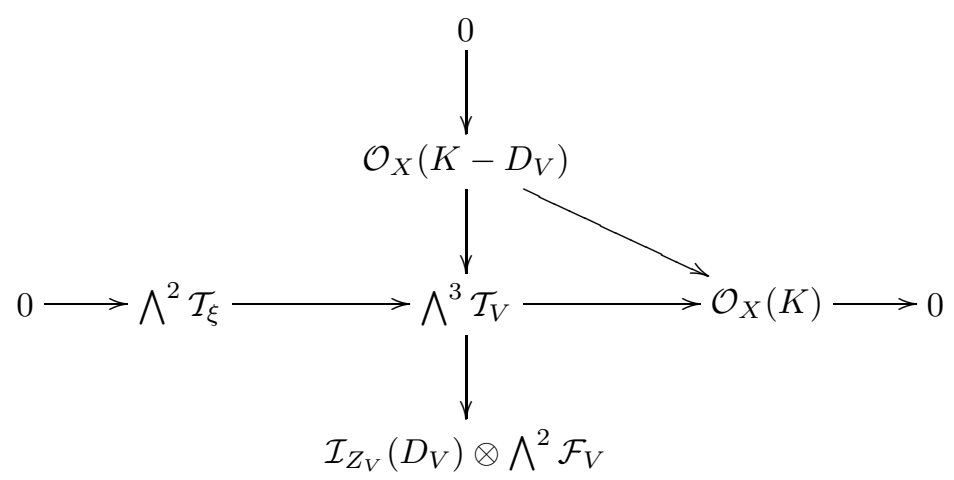

The restriction of the above diagram to any reduced irreducible component $C$ of $D_{V}$ gives a nonzero morphism $\mathcal{O}_{C}\left(K-D_{V}\right) \longrightarrow \bigwedge^{2} \mathcal{T}_{\xi} \otimes \mathcal{O}_{C}=\mathcal{T}_{\xi}^{*}(K) \otimes \mathcal{O}_{C}$. Tensoring with $\mathcal{O}_{X}(-K)$ we obtain a nonzero morphism $\mathcal{O}_{C}\left(-D_{V}\right) \longrightarrow \mathcal{T}_{\xi}^{*} \otimes \mathcal{O}_{C}$. Combining this with the defining sequence for $\mathcal{T}_{\xi}$ yields

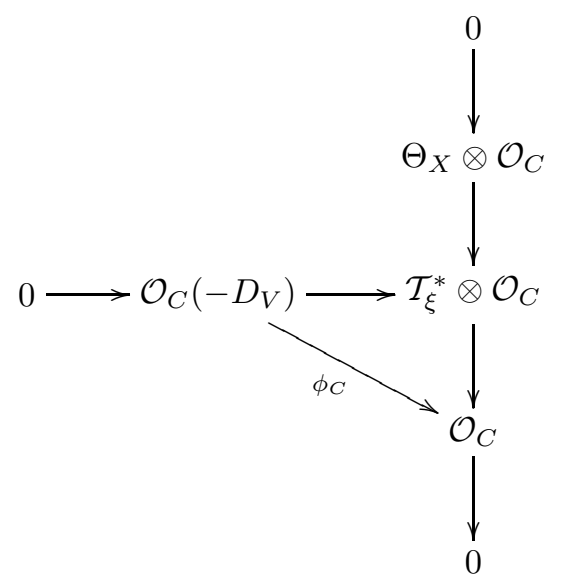

Observe that $D_{V} \cdot C \geq 0$ since otherwise $\phi_{C}=0$ and we obtain a nonzero morphism $\mathcal{O}_{C}\left(-D_{V}\right) \longrightarrow \Theta_{X} \otimes \mathcal{O}_{C}$ which contradicts the ampleness of $\Omega_{X}$. This proves that $D_{V}$ is nef. But $D_{V}$ is a component of $E_{1}$ which implies, by Lemma 5.1 , that $D_{V}^{2} \leq 0$. Thus we must have $D_{V} \cdot C=0$ for every reduced irreducible component $C$ of $D_{V}$. Returning to (5.25) we see that the morphism $\phi_{C}$ must be nonzero and, hence, it is an isomorphism for every reduced irreducible component $C$ of $D_{V}$. This implies, in particular, that $Z_{V}=\emptyset$. 
Once we know that $Z_{V}=\emptyset$ the diagram (5.19) becomes as follows

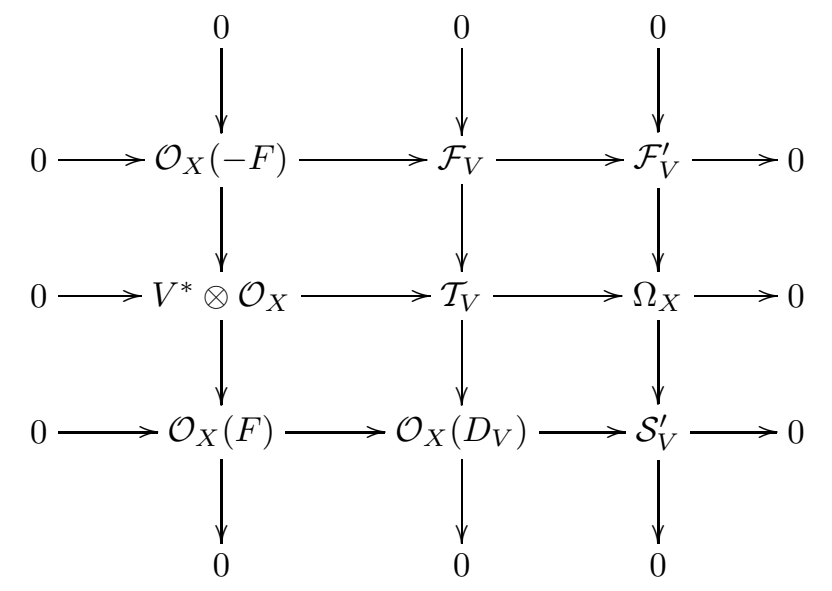

Assume $E_{V}=c_{1}\left(\mathcal{S}_{V}^{\prime}\right)=D_{V}-F \neq 0$. We will show that this produces a nonzero subspace of locally supported moduli which is impossible by the first assertion of the proposition. To this end dualize the sequence at the bottom of the above diagram to obtain

$$
0 \longrightarrow \mathcal{O}_{X}\left(-D_{V}\right) \longrightarrow \mathcal{O}_{X}(-F) \longrightarrow \mathcal{E x t}^{1}\left(\mathcal{S}_{V}^{\prime}, \mathcal{O}_{X}\right) \longrightarrow 0
$$

This implies that $\mathcal{E} x t^{1}\left(\mathcal{S}_{V}^{\prime}, \mathcal{O}_{X}\right)=\mathcal{O}_{E_{V}}(-F)$. Furthermore, since $F_{V}$ is a component of $D_{V}$ we have $D_{V} \cdot F=0=E_{V} \cdot F$ which implies $\mathcal{E} x t^{1}\left(\mathcal{S}_{V}^{\prime}, \mathcal{O}_{X}\right)=\mathcal{O}_{E_{V}}$. Now dualizing the column on the right in (5.26) we obtain

$$
0 \longrightarrow \Theta_{X} \longrightarrow\left(\mathcal{F}_{V}^{\prime}\right)^{*} \longrightarrow \mathcal{O}_{E_{V}} \longrightarrow 0
$$

which yields that $\operatorname{ker}\left(H^{1}\left(\Theta_{X}\right) \longrightarrow H^{1}\left(\mathcal{F}_{V}^{\prime}\right)\right) \neq 0$. But this is a subspace of locally supported moduli which must be zero by the first part of the proof of the proposition. Thus $c_{1}\left(\mathcal{S}_{V}^{\prime}\right)=0$ and we obtain the equality $\mathcal{F}_{V}^{\prime}=\Omega_{X}$.

\section{REFERENCES}

[1] W. Barth, C. Peters, A. Van de Ven, Compact complex surfaces, Ergeb. Math. Grenzgeb, 1984.

[2] A. Beauville, L'application canonique pour les surfaces de type général, Invent. Math., 55 (1979), pp. 121-140.

[3] E. Bombieri, Canonical models of surfaces of general type, Publ. Math. Inst. Hautes Etud. Sci., 42 (1973), pp. 171-219.

[4] E. Calabi, E. Vesentini, On compact locally symmetric Kähler manifolds, Ann. of Math., 71 (1960), pp. 472-507.

[5] F. Catanese, Moduli of algebraic surfaces, in: Theory of Moduli. Proceedings C.I.M.E. 1985 (LNM, Vol. 1337, pp. 1-83) Berlin, Heidelberg, New-York, Springer 1988.

[6] F. Catanese, On the moduli spaces of surfaces of general type, J. Differ. Geom., 19 (1984), pp. $483-515$.

[7] F. CATANese, Moduli and classification of irregular Kähler manifolds(and algebraic varieties) with Albanese general type fibrations, Inven. Math., 104 (1991), pp. 263-289.

[8] Z. Chen, The existence of algebraic surfaces with pre-assigned Chern numbers, Math. Z., 206 (1991), pp. 241-254.

[9] F. Hirzebruch, Arrangements of lines and algebraic surfaces, Arithmetic and Geometry, Vol.II, Progress in Math., Vol. 36, pp. 113-140. Boston, Basel, Stuttgart, Birkhäuser 1983. 
[10] E. HorikaWA, On algebraic surfaces with pencils of genus 2, in Complex Analysis and Algebraic geometry, volume dedicated to Kodaira, pp. 79-90, Cambridge 1977.

[11] J. Lipman, Free Derivation modules on algebraic varieties, American Journal of Math., 87 (1965), pp. 874-898.

[12] Y. MIYAOKA, The maximal number of quotient singularities on surfaces with given numerical invariants, Math. Ann., 268 (1984), pp. 159-171.

[13] Y. MiYAoKA, The Chern class and Kodaira dimension of minimal variety, Adv. Study Pure Math., 10 (1987), pp. 449-476.

[14] Y. MiYaOKA, On the Kodaira dimension of minimal threefold, Math. Ann., 281 (1988), pp. 325-332.

[15] U.Persson, Chern invariants of surfaces of general type, Compositio Math., 43 (1981), pp. $3-58$.

[16] U. Persson, An Introduction to the Geography of surfaces of general type, Proceedings of Sypm. in Pure Math., 46 (1987).

[17] A. Sommese, On the density of ratios of Chern numbers of algebraic surfaces, Math. Ann., 268 (1984), pp. 207-221.

[18] G. XIAO, Surfaces fibrées en courbes de genre deux, LNM 1137, Berlin, Heidelberg, New-York, Springer 1985.

[19] G. XIAO, Fibred algebraic surfaces with low slope, Math. Ann., 276 (1987), pp. 449-466.

[20] G. XIAO, An example of hyperelliptic surfaces with positive index, preprint(1985).

[21] S.-T. YAU, Calabi's conjecture and some new results in algebraic geometry, Proc. Nat. Acad. Sci. USA (1977), pp. 1798-1799. 